\title{
Development of a 5.4 MV laser triggered gas switch for multimodule, multimegampere pulsed power drivers
}

\author{
K. R. LeChien, ${ }^{1}$ M. E. Savage, ${ }^{1}$ V. Anaya, ${ }^{2}$ D. E. Bliss, ${ }^{1}$ W. T. Clark, ${ }^{1}$ J. P. Corley,${ }^{2}$ G. Feltz, ${ }^{2}$ J. E. Garrity, ${ }^{3}$ D. W. Guthrie, ${ }^{2}$ \\ K. C. Hodge, ${ }^{2}$ J. E. Maenchen, ${ }^{1}$ R. Maier, ${ }^{1}$ K. R. Prestwich, ${ }^{4}$ K. W. Struve, ${ }^{1}$ W. A. Stygar, ${ }^{1}$ T. Thompson, ${ }^{2}$ \\ J. Van Den Avyle, ${ }^{1}$ P. E. Wakeland, ${ }^{2}$ Z. R. Wallace, ${ }^{2}$ and J. R. Woodworth ${ }^{1}$ \\ ${ }^{1}$ Sandia National Laboratories, Albuquerque, New Mexico 87185, USA \\ ${ }^{2}$ Ktech Corporation, Albuquerque, New Mexico 87123, USA \\ ${ }^{3}$ Materials Processing and Coatings Laboratory, Albuquerque, New Mexico 87123, USA \\ ${ }^{4}$ Kenneth R. Prestwich Consulting, Albuquerque, New Mexico 87112, USA \\ (Received 28 February 2008; published 18 June 2008)
}

\begin{abstract}
Laser triggered, megavolt, megampere gas switches are frequently utilized to synchronize multiple pulsed power driver modules for inertial-confinement fusion, isentropic compression, and radiation physics experiments. The device developed to synchronize the 36 modules of the refurbished $Z$ accelerator at Sandia National Laboratories is a $5.4 \mathrm{MV}, 700 \mathrm{kA}$, sulfur-hexafluoride $\left(\mathrm{SF}_{6}\right)$ filled, laser triggered gas switch. At this operating level, switch jitter is $5 \mathrm{~ns}$, the prefire rate is less than $0.1 \%$, the average optic lifetime is greater than 200 shots, and the flashover rate is less than $1 \%$. Over 1000 shots on a single-module test facility were conducted while iterating several potential design improvements, including utilizing low-erosion electrode material, varying $\mathrm{SF}_{6}$ pressure, and modifying internal switch geometry all while keeping the basic switch architecture and footprint constant. Results of this development effort are presented herein.
\end{abstract}

DOI: 10.1103/PhysRevSTAB.11.060402

PACS numbers: $51.50 .+\mathrm{v}, 52.75 . \mathrm{Kq}$

\section{INTRODUCTION}

Multimegampere pulsed power drivers for inertialconfinement fusion, isentropic compression, radiation physics, and equation-of-state studies require robust and reliable switching. Petawatt class $z$-pinch accelerators require nanosecond synchronization of multiple pulsed power drivers each operating at the 5-6 MV, $1 \mathrm{MA}$ level [1]. In many multimodule machine architectures, energy is capacitively stored typically in a series erecting Marx bank. Each Marx stage is switched at low voltage $(\sim 200 \mathrm{kV})$ into intermediate storage capacity with a rise time of order $\sim 1 \mu \mathrm{s}$ (self-limited by Marx capacitance and inductance). Several stages of pulse transmission and compression are necessary to achieve petawatt electrical and $\mathrm{x}$-ray powers into loads of interest.

There are several options for the first stage of pulse compression for a multimegavolt system with a long charge time $(\sim 1 \mu \mathrm{s})$ when nanosecond synchronization is required. They are (i) liquid dielectric switching, (ii) solid dielectric switching, (iii) vacuum dielectric switch, and (iv) gas dielectric switching. Liquid switching requires long, highly resistive gaps, and a high-energy laser triggering system [2]. A desire for a low-maintenance switch precludes the use of solid dielectric switching since each machine discharge results in destruction of the solid switch. Vacuum switching requires large insulation envelopes that are necessarily highly inductive. In addition, a significant research effort is required to develop multimegavolt liquid, solid, or vacuum switches and their triggering systems before they could be reliably utilized for this application.
The first stage of pulse compression for a multimodule system is best suited for high pressure gas switches. Examples of multimegavolt, multimodule, multimegampere systems that utilize gas switching as the last stage of command triggered power flow include Proto II [3] (16 switches, electrically triggered), Double EAGLE [4] (2 switches, electrically triggered), PBFA I [5] (36 switches, electrically triggered), HERMES III [6] (20 switches, laser triggered), SATURN [7] (36 switches, electrically triggered), PBFA II [8] (36 switches, electrically triggered), $Z$ [9] (36 switches, laser triggered), and the refurbished $Z$ accelerator [10] (36 switches, laser triggered).

There are generally three methods for triggering a gas switch. They are (i) self-closure switching, (ii) electrical triggering, and (iii) laser triggering. Self-closure switching, or allowing a gas switch to overvolt with no command fire, is not reliable for nanosecond jitter and is therefore not feasible for a petawatt multimodule system. Electrical triggering, or overvolting a pressurized gap by the application of a high-voltage pulse to initiate streamer closure, requires a provision for inductive isolation of the trigger generator to the system. A low-loss, megavolt isolation inductor is generally not compact. Laser triggering, or focusing a laser beam to initiate plasma formation, is presently the most reliable way to achieve nanosecond jitter utilizing a compact, off-the-shelf package. Synchronization of these systems requires robust and reliable switching whether the operational mode is for maximum energy efficiency in early time (perfect synchronization of all lines) or for pulse shaping (delayed timing of lines to provide specific pulse shape). Future discussions of 
triggering gas switches throughout this manuscript will focus on laser triggered gas switches (LTGS).

It is the gas switch for these large accelerators that frequently constrains the reliable operation, performance, and maintenance of the system. There is a tradeoff between increasing the size of the switch to operate in the megavolt regime while minimizing the inductance, which are contradictory requirements. (As size increases, the inductance increases and so does the required operating voltage to drive a desired output power). There are generally four major issues that affect the performance of a gas switch. For a multimodule machine these issues, listed in order of relative importance are (i) prefires, or switch closure before command fire, (ii) jitter, defined as statistical spread in closure time, (iii) insulator flashover, or random failure of insulating surfaces, and (iv) degradation of limited life components, such as optics and electrodes.

Arc-channel heating will liberate electrode material causing several failure modes such as prefire, high jitter, and flashover. For example, liberation of electrode material in the form of large (micrometer in size) metal particulate may coat insulator surfaces that increases the probability of insulator flashover. Insulator flashover may or may not affect a machine shot, but the probability of subsequent failure of that insulator surface is dramatically increased, ultimately resulting in a prefire. Liberation of electrode material in the form of a fine powder (nanometer in size) can coat optics effectively blocking the laser beam path, rendering useless a command fire. Generally, the lifetime of a switch will be defined by the extent to which these issues collaborate, the impact of which may be lessened by designed mitigations for each of the issues. The desire, of course, is to operate a LTGS with some predefined and known lifetime.

Prefire rate and jitter are coupled parameters by the chosen operating pressure and gas type. Sulfurhexafluoride $\left(\mathrm{SF}_{6}\right)$ is commonly utilized as the fill gas for megavolt applications because of its high dielectric strength at pressures of a few atmospheres. The pulsed dielectric strength of $\mathrm{SF}_{6}$ increases as the pressure is increased to some threshold [11]. Therefore, for a given operating voltage the pressure may be increased to that threshold to reduce the statistical likelihood of a prefire. Voltage jitter, however, also increases with increasing gas pressure in systems where the electrode condition varies. A typical philosophy is to find the highest operating pressure at which the jitter of a switch is acceptable, and at this empirically discovered pressure the switch should have the lowest prefire rate. This generally happens to be between $75 \%-85 \%$ of self-closure voltage at the operating pressure. It is important to stress that it is not possible to choose a prefire rate and jitter as if they are separable quantities.

Previously published manuscripts discussing multimegavolt gas switches frequently quote a maximum operating voltage for the device. Typically these devices achieved this voltage for a limited number of self-closure tests, but acceptable performance could not be demonstrated at this level with reasonable reliability. Examples of manuscripts that quote maximum achieved voltages as reliable operating points include switches designed for 2.3 MV [12], 2.4 MV [13,14], 2.8 MV [15], 3 MV [4], 3 MV [16], $4 \mathrm{MV}$ [17], $5 \mathrm{MV}$ [18], $5 \mathrm{MV}$ [19], $6 \mathrm{MV}$ [20], $6 \mathrm{MV}$ [8], $6 \mathrm{MV}$ [21], and $6 \mathrm{MV}$ [22-24]. Each of these switches did not perform in excess of $76 \%$ of the quoted maximum value for a statistically significant number of machine shots. To the authors' knowledge, there are no reviewed journal articles on the development of a switch that has a reliable operating point for an acceptable machine lifetime in excess of five megavolts.

This manuscript summarizes the development of a LTGS that has a reliable operating point at $5.4 \mathrm{MV}$ and $700 \mathrm{kA}$ for the state-of-the-art refurbished $Z$ facility [10]. The entire $Z$ pulsed power system was replaced between July 2006 and September 2007 to improve reliability and increase energy delivered to the load. A cross section of the upgraded driver is depicted in Fig. 1. The pulsed power system delivers more than nine megajoules of forwardgoing wave energy in the first 100 nanoseconds of its pulse. The system is comprised of 36 nominally identical modules, each producing a 3.3-terawatt pulse in $6 \Omega$ waterinsulated transmission lines. The peak forward-going voltage is $\sim 5 \mathrm{MV}$. The $10 \%-90 \%$ forward wave pulse rise time is $\sim 75 \mathrm{~ns}$; the full width at half maximum is $\sim 190 \mathrm{~ns}$. The 36 modules are combined in parallel and drive 20 to $25 \mathrm{MA}$ into the single load.

The 5.4 MV LTGS discussed in this manuscript discharges the intermediate store capacitor and it is the final command triggered switch in the system. This switch must withstand 5.4 MV before triggering, and conduct $700 \mathrm{kA}$ after closure for near-term machine requirements. The

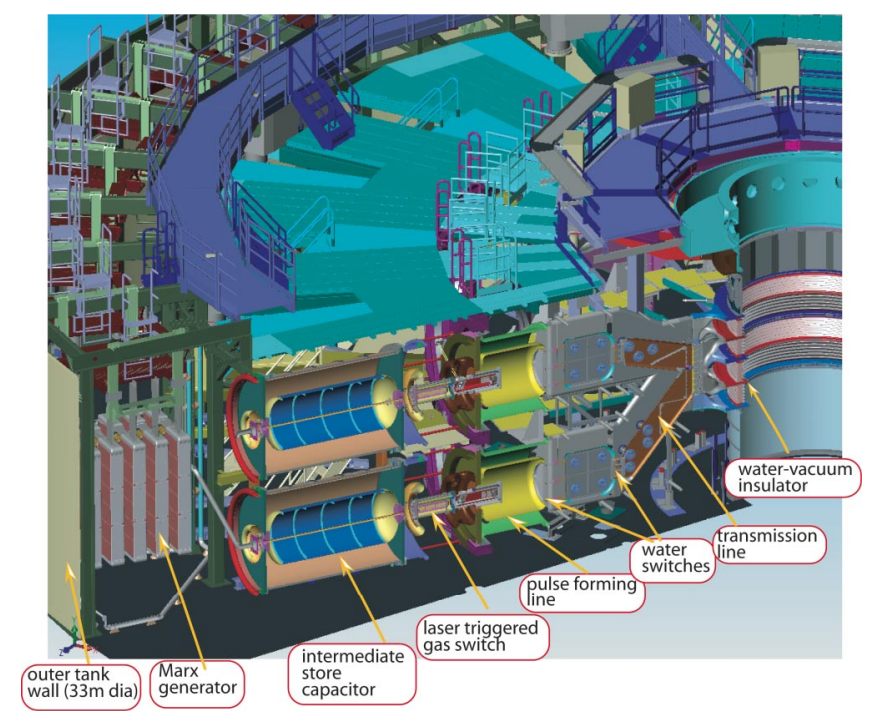

FIG. 1. (Color) Cross section of the refurbished $Z$ driver. The outer tank wall is $33 \mathrm{~m}$ in diameter. 
closure of the gas switch initiates charging of the pulseforming line. The output switches for the pulse-forming line are self-closing water switches [25,26]. The end of this line has an additional set of water switches with a primary purpose of reducing the prepulse from capacitive coupling across the main water switch gaps. After the second water switch gaps, power flows through passive water lines to the water convolute. The water convolute splits power across the four levels of the water vacuum interface [27]. Power flows on magnetically insulated transmission lines to a posthole convolute to the load.

Performance requirements for the LTGS for the refurbished driver are given in Sec. II. A description of the comprehensive research effort that began in August 2005 to deliver an acceptable LTGS is given in Sec. III. An overview of the 5.4 MV LTGS is given in Sec. IV, with detail given for the operation of each portion of the device in Secs. IVA and IV C. An overview of the laser triggering system for the LTGS that was developed for the refurbished $Z$ is given in Section IVB 2. A description of advanced cleaning and handling protocols that were developed as part of the research effort is given in Sec. V. A summary of the results from this effort as well as future work is given in Sec. VI.

\section{PERFORMANCE REQUIREMENTS FOR THE LTGS}

The shot-rate goal for the refurbished $Z$ accelerator is two shots per day, double the shot rate of the accelerator before the refurbishment [28]. Prefires, late fires, and other switch failures must be less than $0.03 \%$ for an individual switch to meet the requirement of less than $1 \%$ total machine failures caused by the LTGS.

The requirement for maximum shot-to-shot jitter, $\sigma_{T}$, of the forward-going wave for the 36 modules is $1 \mathrm{~ns}$ since aspects of load behavior occur in $\sim 1$ ns time scales. Fast diagnostics, such as Z-Beamlet [29], are synchronized with machine output current for load energy and density measurements. Therefore, the maximum jitter for an individual module $\sigma_{M}$ goes as

$$
\sigma_{M}=\sqrt{n} \sigma_{T}
$$

assuming a normal distribution, where $n$ is 36. Jitter in the forward-going wave is dominated by LTGS jitter. Therefore, the jitter of the gas switch must be no larger than $6 \mathrm{~ns}$, over some well-defined lifetime. The long term jitter goal is $0.2 \mathrm{~ns}$ for femtosecond laser ignition inertialconfinement-fusion (ICF) experiments and will require a second-generation LTGS with an individual jitter of $\sim 1$ ns. Future switch improvements are discussed in Sec. VI.

The capacitively stored energy was doubled during the refurbishment from $11.4 \mathrm{MJ}$ to $22.8 \mathrm{MJ}$ at a $90 \mathrm{kV}$ charge. The goal for peak machine current at a $90 \mathrm{kV}$ charge in $100 \mathrm{~ns}$ into a $20 \mathrm{~mm}$ radius, $20 \mathrm{~mm}$ long wire array load is
TABLE I. Goals, requirements, and switch performance at 5.4 MV for the LTGS developed for the refurbished $Z$ accelerator.

\begin{tabular}{lccc}
\hline \hline & $\begin{array}{c}\text { Gas switch } \\
\text { goals }\end{array}$ & $\begin{array}{c}\text { June 2005 } \\
\text { performance }\end{array}$ & $\begin{array}{c}\text { Present gas } \\
\text { switch } \\
\text { performance }\end{array}$ \\
\hline Volameter & $6.3 \mathrm{MV}$ & $5-6.25 \mathrm{MV}$ & $5.4 \mathrm{MV}$ \\
Prefire rate & $<0.03 \%$ & $\sim 8 \%$ & $<0.1 \%$ \\
Jitter (ns) & $<6 \mathrm{~ns}$ & $13 \mathrm{~ns}$ & $5 \mathrm{~ns}$ \\
Optics life & $>200$ shots & $\sim 35$ shots & $\sim 800$ shots \\
Flashover rate & $0.03 \%$ & $\sim 5 \%$ & $<1 \%$ \\
Replacement interval & $>100$ shots & $<30$ shots & $>100$ shots \\
\hline \hline
\end{tabular}

$21 \mathrm{MA}$. A LTGS voltage of $5.4 \mathrm{MV}$ is required to drive this current in $100 \mathrm{~ns}$ [30].

Failures, such as flashover and prefire, result from deterioration of electrodes, optics, and insulators which are shot-dependent failure modes. As the constituents of a switch degrade the jitter performance is negatively affected. Prefires may also result from other contributing factors, including random conditions, such as an uncharacteristically poor electrode condition or unfavorable electrode material deposition on insulators. Design tradeoffs were implemented that reduced the affects of a lowprobability occurrence at the expense of shortening the average lifetime (trading better defined standard deviation for shorter lifetime). The goal for the lifetime of an individual LTGS is 150 shots without maintenance. The standard deviation in lifetime should be less than $5 \%$, since it is the variance in lifetime that dictates a planned maintenance schedule. All switch design aspects favored a well-defined standard deviation in switch lifetime over increasing the average switch lifetime, if there was a design tradeoff between the two.

LTGS performance at several stages of development is summarized in Table I. A comprehensive research program to improve LTGS performance to meet the refurbished accelerator performance goals was initiated in August 2005. This research effort elevated gas switch design to previously unexplored physical regimes and led to the need for extremely well-controlled experiments in a terawatt environment. The results of that effort are presented herein.

\section{RESEARCH EFFORT TO MEET PERFORMANCE GOALS}

The laser triggered gas switch that is designed for the refurbished $Z$ accelerator is an evolution of the Rimfire gas switch first implemented on Hermes III [13]. A description of this switch is given elsewhere [22]. Voltage and current requirements increased $25 \%$ from the onset of the design effort of the LTGS with no allowable increase to the physical footprint or inductance of the device. Initial design studies indicated that a total machine peak current of 26 MA into loads of interest could be achieved with each 
LTGS operating at $5 \mathrm{MV}$ and $600 \mathrm{kA}$. Increases in the inductance of the transition from vertical watertransmission lines to horizontal magnetically insulatedtransmission lines, higher inductance in vacuum from changes in the load position for improved diagnostic access, and conservatism in the vacuum power flow requirements caused the LTGS operating requirements to become 5.4 MV and $700 \mathrm{kA}$ for a total machine peak current of $21 \mathrm{MA}$ into a $20 \mathrm{~mm}$ radius, $20 \mathrm{~mm}$ in length single wirearray load.

The design effort was conducted on an engineering module called $Z_{20}$ that replicates one module of the refurbished $Z$ accelerator from the Marx to the interface with the water convolute [31]. Initial experiments from the originally designed LTGS for the refurbishment of the $Z$ accelerator were conducted over a voltage range of $5.5 \mathrm{MV}$ to $6.3 \mathrm{MV}$ with a switch operating pressure in excess of 4.15 bar [50 psi (gauge)]. Under these conditions the switch jitter was $13 \mathrm{~ns}$, the prefire rate was $\sim 8 \%$, the average lifetime of the final optic assembly was 36 shots, and the flashover rate was $\sim 5 \%$. All of these performance parameters did not adequately meet the requirements of the LTGS for the refurbished $Z$ accelerator.

We identified several switch design issues that were addressed to improve reliability. They are (i) reducing the peak electric field and reducing electric field nonuniformities in the trigger gap, (ii) identifying a robust trigger material for megampere pulsed current operation, (iii) tailoring self-closing cascade gaps to reduce the peak field and achieve axial field uniformity, (iv) specifying all electrode and insulator materials in addition to preparation and cleaning procedures, and (v) electrically shielding metal-plastic-gas triple points. Each item was integral in improving performance to meet the requirements of the LTGS for the refurbished $Z$ accelerator. These improvements are summarized in the following sections.

\section{SWITCH DESIGN}

The LTGS depicted in Fig. 2 is comprised of two series switches; a laser triggered portion, and a self-closing portion, called the cascade portion. The necessity for this topology is based on physical size requirements to insulate 5.4 MV utilizing $\mathrm{SF}_{6}$, and on the availability of compact ultraviolet lasers that are appropriate for laser triggering in $\mathrm{SF}_{6}$. The voltage impressed across the trigger gap during charging accounts for $14.9 \%$ of the total voltage across the switch. The trigger and cascade sections share the same volume of $\mathrm{SF}_{6}$ gas. Therefore, the respective operating electric fields and pressures must not be significantly mismatched otherwise the switch will not be optimized for either low prefire rate or low jitter. Computer calculation and experiment on switches of similar design have been conducted over the past several years [23,24,32-35].

Prefire probability and switch jitter are coupled parameters by the operational electric field and pressure. We have

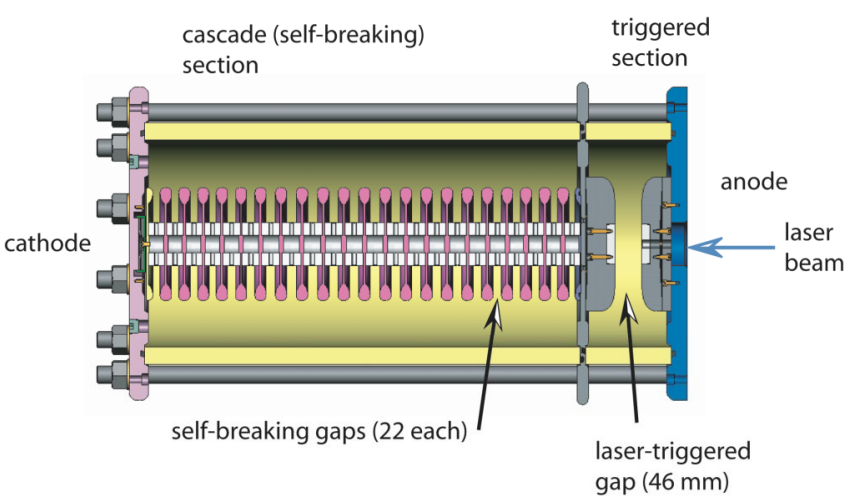

FIG. 2. (Color) Cross section of the 5.4 MV LTGS utilized in the refurbished $Z$ accelerator.

found that applying Weibull reliability statistics to selfclosure voltage data is very effective in determining the extent of this coupling, i.e., what pressure is appropriate for a given voltage for some desired prefire rate. It is both the spread in self-closure voltage and the average self-closure voltage for a given pressure that determines the operating point of a switch. From these analyses, it was possible to optimize the trigger geometry and significantly improve trigger section performance. A discussion of prefire rate is given in Sec. IV B 1 and discussion of trigger jitter is given in Sec. IV B 2.

\section{A. Trigger and cascade electrode material testing and observed failure modes at $5.4 \mathrm{MV}$ and $700 \mathrm{kA}$}

At 5.4 MV the switch peak current is $700 \mathrm{kA}$ with approximately $0.1 \mathrm{C}$ of charge transferred in the first $200 \mathrm{~ns}(\sim 1 \mathrm{C}$ total per pulse); the action integral value is $6 \times 10^{4} \mathrm{~A}^{2} \mathrm{~s}$. The trigger gap has a single arc channel with a current density of $\sim 10^{7} \mathrm{~A} / \mathrm{cm}^{2}$ while each cascade gap has five arc channels on average to distribute this current over a larger area. Energy in the form of heat and magnetic pressure is deposited both into the surface of the electrode and the bulk material. This deposition explosively disperses electrode material (i.e. erosion), causes liquid droplet ejection, and viscous shearing of molten liquid [36-38]. How exactly the material ablates under high temperature and pressure conditions such as by fracturing or ejecting fine powder will exhibit unique failure modes. Electrode erosion will to some degree increase prefire rate, increase flashover rate, and/or increase optics degradation rate. Other switch operating conditions, such as gas pressure, affect the extent to which electrode erosion manifests into these failure modes [39].

The rate at which an electrode erodes depends on its material properties including melting temperature, latent heat of vaporization, thermal shock resistance, and electrical conductivity [40,41]. Refractory metals are generally utilized as the base metal for electrodes in high temperature applications because of their high melting temperature $[42,43]$. Typically a refractory metal is combined with a 
TABLE II. Summary of electrode materials tested and their characteristics.

\begin{tabular}{|c|c|c|c|c|c|c|c|c|}
\hline Material & Composition & Microstructure & $\begin{array}{l}\text { Average } \\
\text { grain } \\
\text { size } \\
(\mu \mathrm{m})\end{array}$ & Processing & $\begin{array}{l}\text { Thermal } \\
\text { conductivity } \\
(\mathrm{W} / \mathrm{m}-\mathrm{K})\end{array}$ & $\begin{array}{l}\text { Electrical } \\
\text { resistivity } \\
(\mu \Omega-\mathrm{cm})\end{array}$ & $\begin{array}{l}\text { Melting } \\
\text { temperature } \\
\text { (C) }\end{array}$ & $\begin{array}{l}\text { Density } \\
(\mathrm{g} / \mathrm{cc})\end{array}$ \\
\hline Cres 304L & $\begin{array}{l}\text { ASTM } \\
\text { A240 }\end{array}$ & $\begin{array}{l}\text { Homogeneous grains with } \\
\text { sulfur rich lamellar phases }\end{array}$ & NA & Unknown & 16.2 & 72 & 1400 & 8 \\
\hline Molybdenum & $\begin{array}{l}\text { ASTM } \\
\text { B387, } \\
\text { type } 361\end{array}$ & Elongated grains & NA & Unknown & 138 & 5.7 & 2617 & 10.22 \\
\hline HD-17 & $\begin{array}{l}90 \% \mathrm{~W} \\
6 \% \mathrm{Ni} \\
4 \% \mathrm{Cu}\end{array}$ & $\begin{array}{l}\text { Coarse spherical } \mathrm{W} \text { particles in } \mathrm{Cu} \text { matrix, } \\
\text { minimal sintering, minimal intergranular } \\
\text { necking between } \mathrm{W} \text { particles }\end{array}$ & $20-50$ & $\begin{array}{l}\text { Liquid } \\
\text { phase } \\
\text { sintered }\end{array}$ & 96 & 12 & $\begin{array}{c}1085(\mathrm{Cu}) \\
3422(\mathrm{~W})\end{array}$ & 17 \\
\hline CW70E & $\begin{array}{l}68 \% \mathrm{~W} \\
32 \% \mathrm{Cu}\end{array}$ & $\begin{array}{l}\text { Fine spherical } \mathrm{W} \text { particles in } \mathrm{Cu} \text { matrix, } \\
\text { large fraction of } \mathrm{W}-\mathrm{W} \text { sintering } \\
\text { and large necking regions }\end{array}$ & $2-7$ & Infiltrated & 210 & 3.6 & $\begin{array}{l}1085(\mathrm{Cu}) \\
3422(\mathrm{~W})\end{array}$ & 13.8 \\
\hline Mallory 1000 & $\begin{array}{l}90 \% \mathrm{~W} \\
6 \% \mathrm{Ni} \\
4 \% \mathrm{Cu}\end{array}$ & $\begin{array}{l}\text { Coarse spherical } \mathrm{W} \text { particles in } \mathrm{Cu} \text { matrix, } \\
\text { small intergranular necking between } \\
\mathrm{W} \text { particles }\end{array}$ & $10-30$ & Infiltrated & 93 & 12 & $\begin{array}{c}1085(\mathrm{Cu}) \\
3422(\mathrm{~W})\end{array}$ & 16.9 \\
\hline
\end{tabular}

higher conductivity metal, such as copper, to improve erosion characteristics further [44]. Materials tested in this research effort include molybdenum, stainless steel 304L, Mallory 1000, HD-17, and CW70E. Their properties are summarized in Table II.

These materials were tested in the required switch geometry, at 5.4 MV, 2.75-2.89 bar, $700 \mathrm{kA}$ in the engineering test module, $Z_{20}$. All tested materials were prepared as in Sec. V. Each switch was tested at full voltage (5.4 MV) until 100 shots or switch failure. Then they were tested in trigger section self-closure mode with the cascade section electrically shorted (approximately $1 \mathrm{MV}$ across only the trigger section). This allowed obtaining prefire statistical data after an either successful test run or a failed test run, either is worst case from a switch lifetime perspective.

A variety of trigger section failure modes were observed that were material dependent. Each trigger electrode material, with the exception of $\mathrm{CW} 70 \mathrm{E}$, led to a switch performance failure that would not meet requirements of the refurbished $Z$ accelerator. Stainless steel 304L, with a melting temperature of $1400 \mathrm{C}$, spalled due to joule heating creating protrusions from the electrode surface evident in Fig. 3 that led to prefires. Molybdenum arc by-products reacted with disassociated $\mathrm{SF}_{6}$ and coated insulators leading to catastrophic flashover in 43 shots. Severe fracturing evident in Fig. 4 also supports this notion. HD-17 fractured depositing $\sim 50 \mu \mathrm{m}$ debris on insulator housings that caused flashing after a dense accumulation is present. Figure 5 shows the $\sim 50 \mu \mathrm{m}$ tungsten particulate becoming freed from the sintered copper matrix in the cross section. The average switch life was 45 shots on three 100-shot-run attempts at the 5.4 MV level with HD-17 trigger electrodes. Tungsten particles from the Mallory
1000 material that are 5-10 nanometers in diameter were ejected from the matrix and reacted with the approximately $5 \mathrm{ppm}$ of moisture in the $\mathrm{SF}_{6}$ gas. This is consistent with the particulate size depicted in Fig. 6. A tungsten-oxide $\left(\mathrm{WO}_{3}\right)$ powder was created that coated optics. When the UV laser beam was incident on the final optic it reacted with the oxide and created a reflective coating eventually rendering it useless. CW70E was the only material that survived a qualifying 100-shot experimental run with no failures. A cross section of this material is depicted in Fig. 7. A summary of failure modes for different trigger electrode materials is depicted in Table III.

Tests were simultaneously conducted on cascade section electrodes manufactured from stainless steel alloy 410 (a martensitic) and alloy 304L (an austenitic). Alloy 410 is hardened steel, and 304L is more ductile. Each arc in the multichanneling cascade section has a current density of $\sim 180 \mathrm{kA} / \mathrm{mm}^{2}$. The brittle nature of the hardened steel fractured when rapidly heated, which deposited electrode material on the cascade insulator leading to an insulator flashover rate of $\sim 12 \%$ at $5.4 \mathrm{MV}$, compared to an insulator flashover rate of $<1 \%$ with alloy 304L. All flashes occurred at bottom dead center of the switch as installed, which is the most likely location for large debris to reside. There were also distinctly different damage patterns on the acrylic housings that were flashed using 410. Chips were missing from discreet locations indicating the flash originated at a localized debris spot.

A comparison of scanning electron microscope cross sections between alloy 410 and alloy 304L at similar arcdamage spots is depicted in Fig. 8. The craters for alloy 410 are approximately $20 \mu \mathrm{m}$ deep and the remaining material is porous. The craters for alloy $304 \mathrm{~L}$ are as much as $8 \mu \mathrm{m}$ 

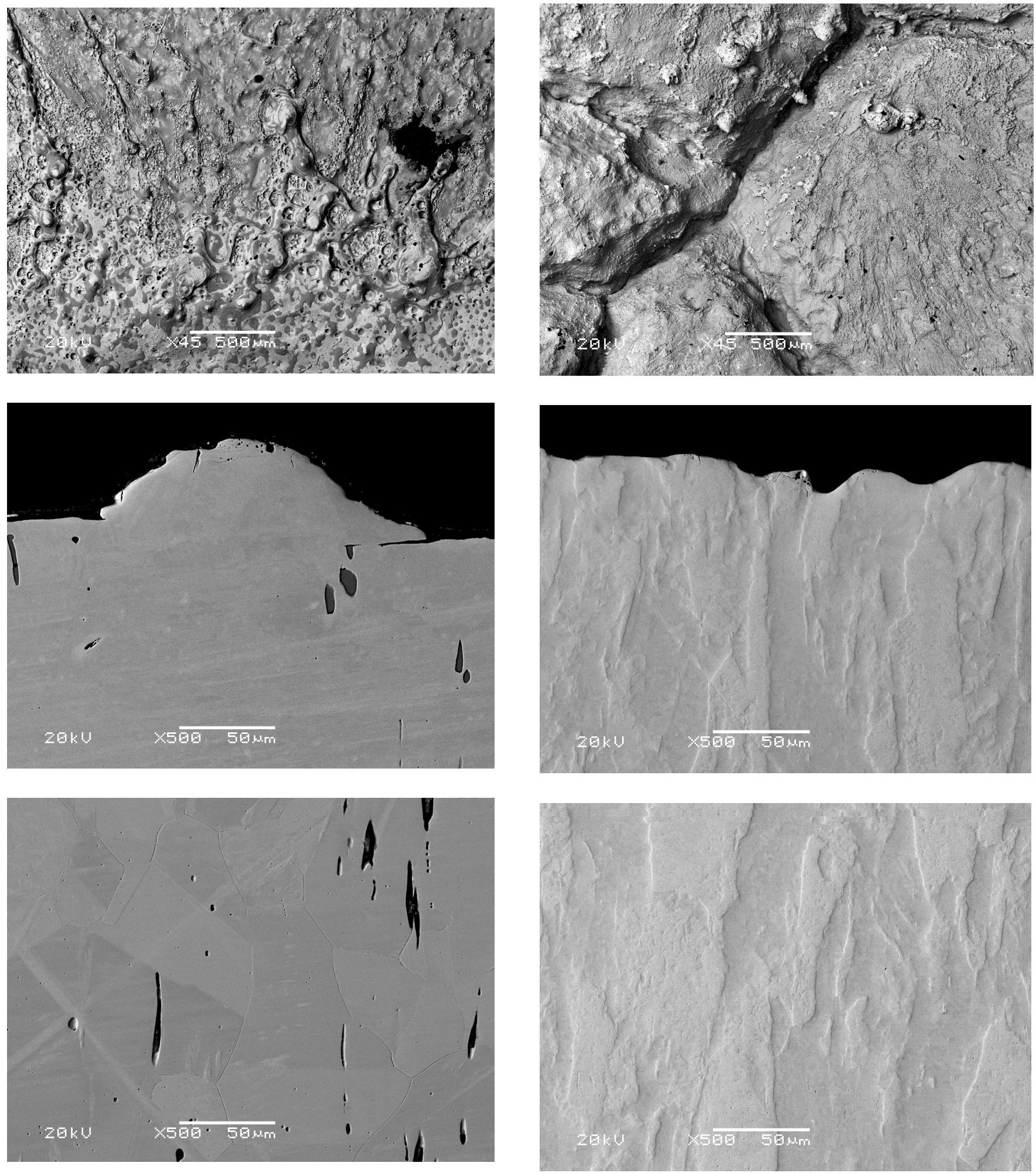

FIG. 3. Backscattered electron micrograph for stainless steel alloy 304L. The top picture is a surface picture of an arc region. The middle picture is a cross section of an arced region. The bottom picture is a cross section of the bulk material.

FIG. 4. Backscattered electron micrograph for molybdenum. The top picture is a surface picture of an arc region. The middle picture is a cross section of an arced region. The bottom picture is a cross section of the bulk material. 

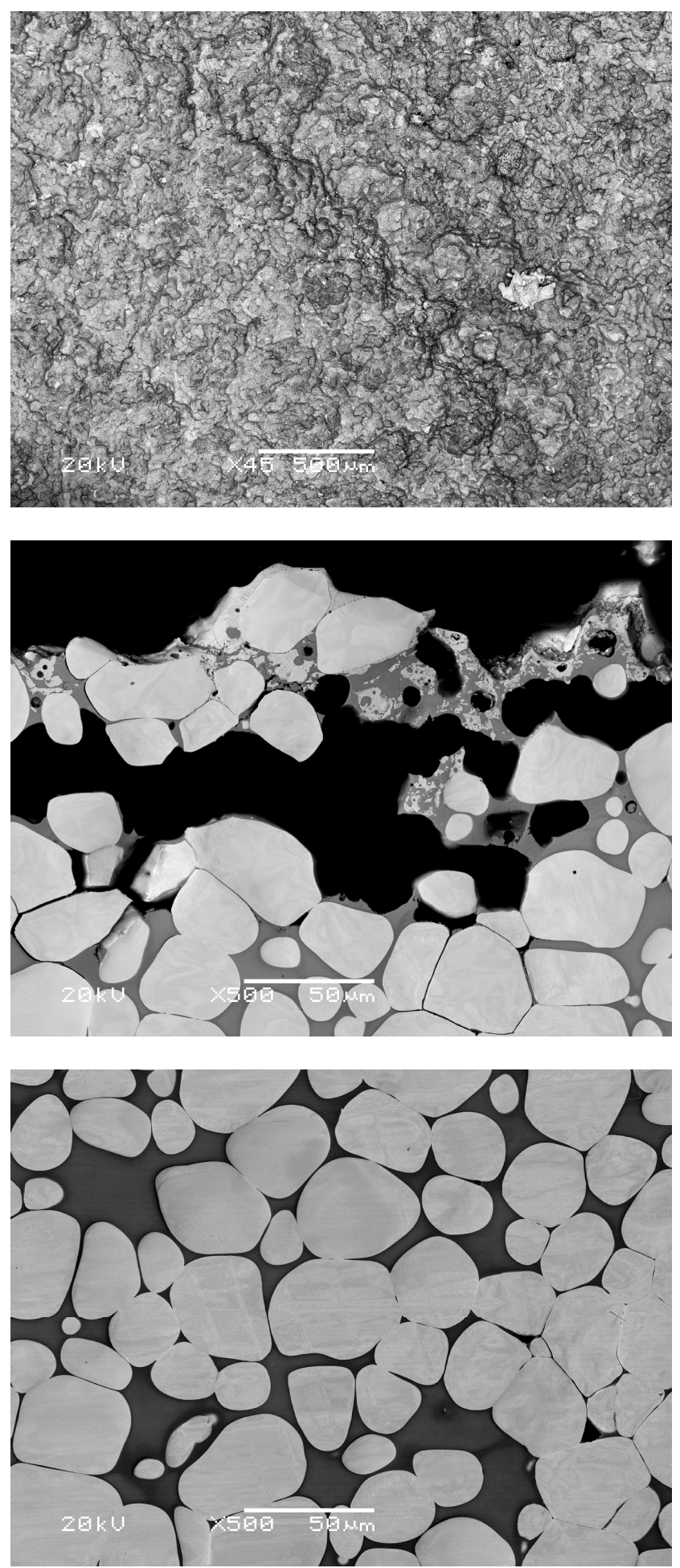

FIG. 5. Backscattered electron micrograph for HD-17. The top picture is a surface picture of an arc region. The middle picture is a cross section of an arced region. The bottom picture is a cross section of the bulk material.
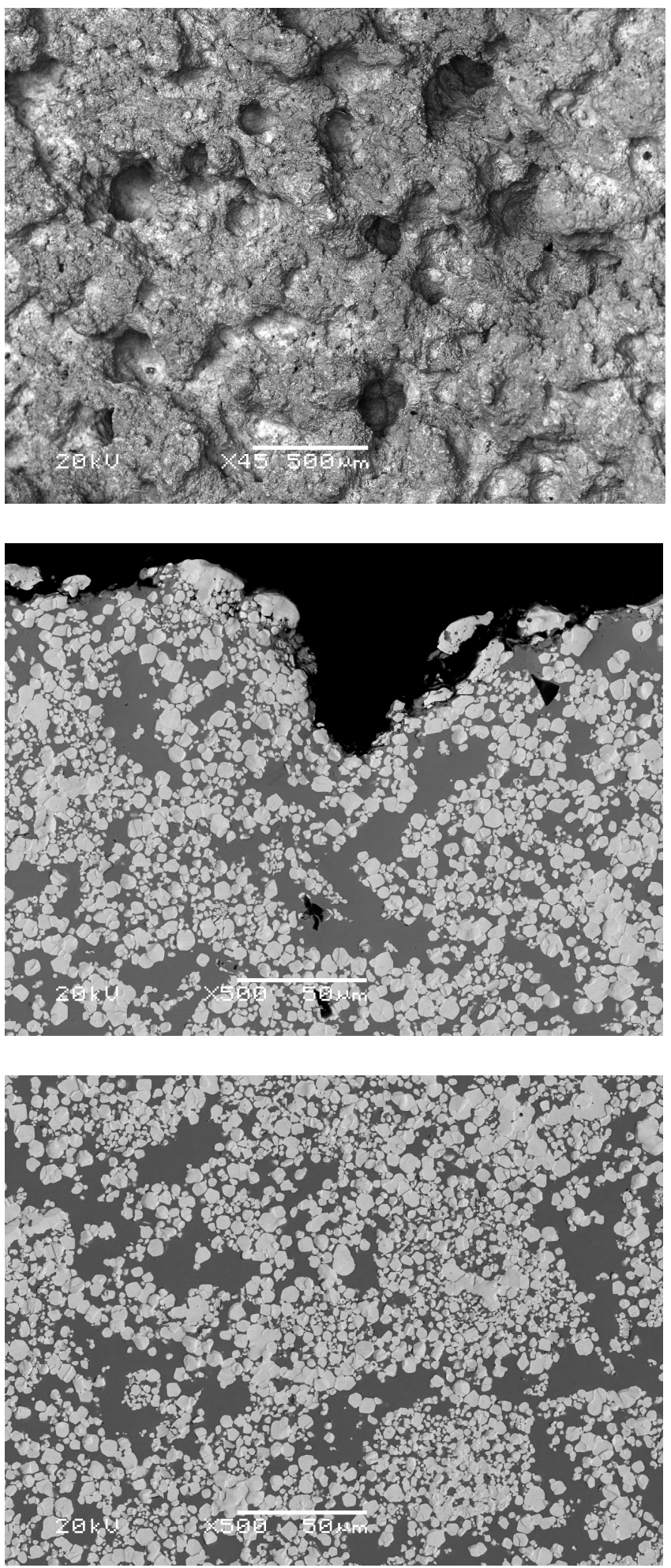

FIG. 6. Backscattered electron micrograph for Mallory 1000. The top picture is a surface picture of an arc region. The middle picture is a cross section of an arced region. The bottom picture is a cross section of the bulk material. 

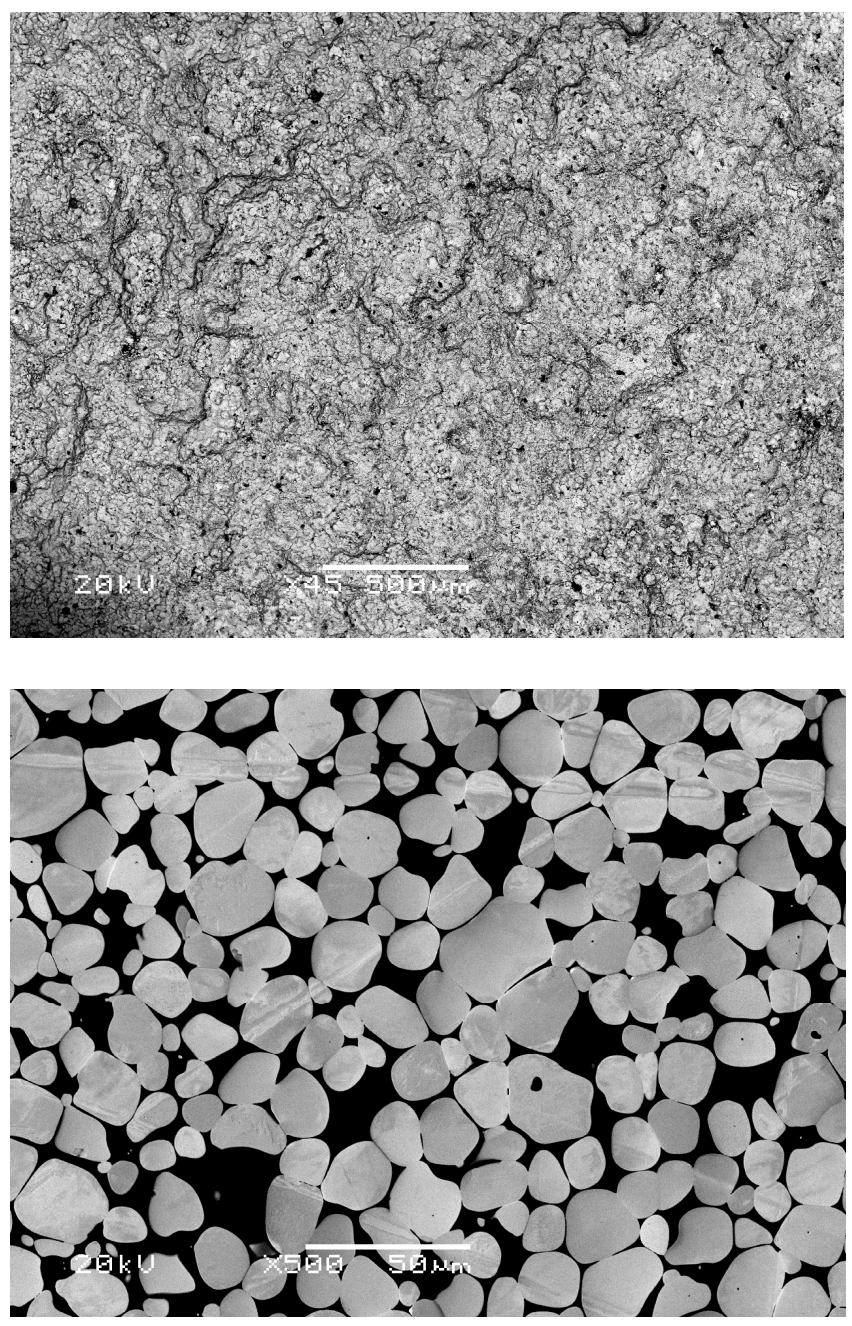

FIG. 7. Backscattered electron micrograph for CW70E. The top picture is a surface picture of an arc region. The bottom picture is a cross section of the bulk material.

deep for one sample. This indicates that the more ductile $304 \mathrm{~L}$ is less likely to liberate large particulates, as noted in the much lower flashover rate of the cascade section insulator when utilizing 304L.

\section{B. Trigger section design and performance}

\section{Self-closure performance}

The trigger electrode profile was hemispherical in shape in the initial design of the LTGS for the refurbished $Z$ accelerator [22]. This geometry is inherently flawed in that conduction current is restricted to a small area $\left(\sim 1 \mathrm{~cm}^{2}\right)$ that is collocated with the highest electric field enhancement as depicted in Fig. 9. This can only lead to varying electrode enhancement with each discharge, and therefore, an opportunity for erratic behavior that is shot dependent.

Self-closure experiments for hemispherical electrodes comprised of HD-17 $(90 \% \mathrm{~W}, 6 \% \mathrm{Ni}, 4 \% \mathrm{Cu}$, liquid sintered, manufactured by Mi-Tech Metals) were conducted at $\mathrm{SF}_{6}$ pressures of 2.75 bar [42 psi (absolute)
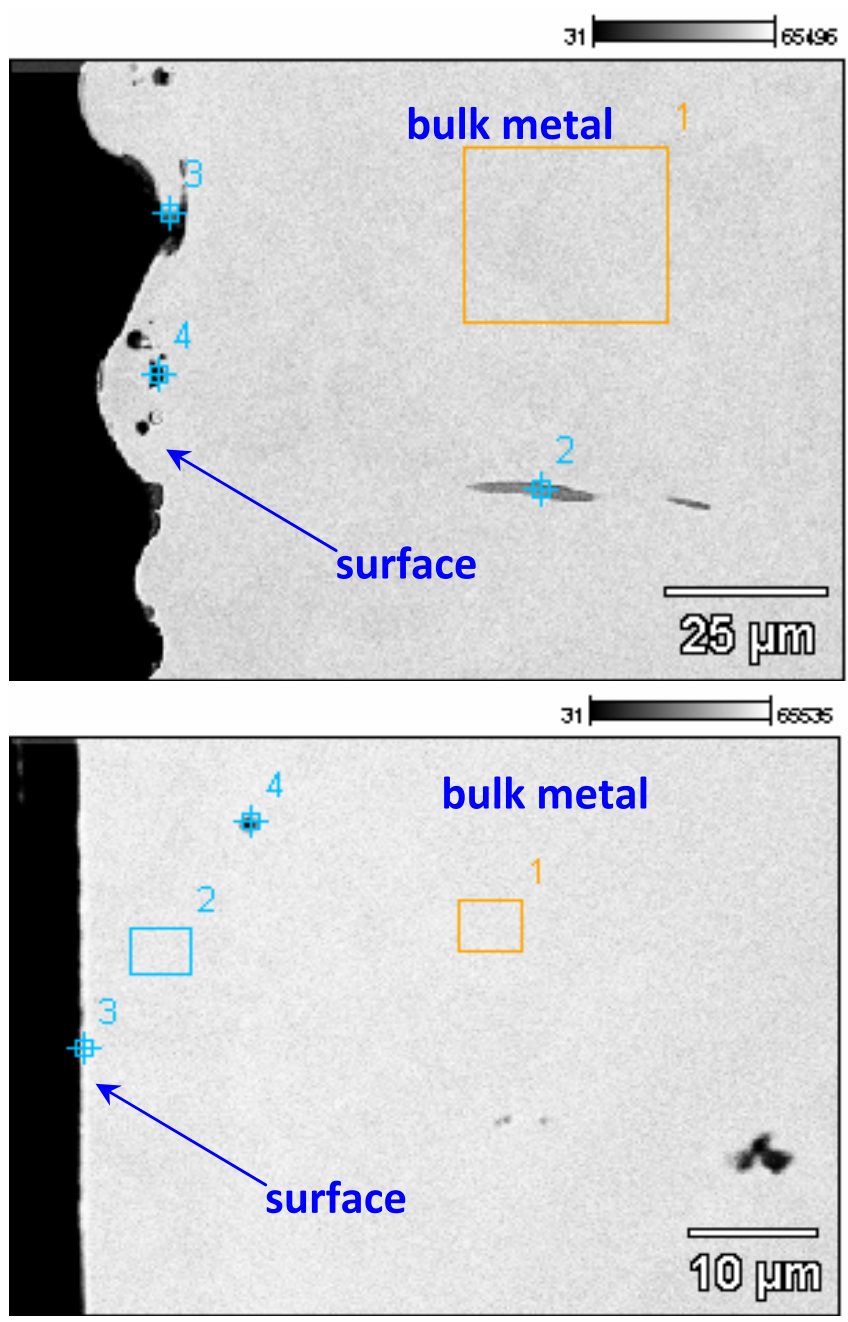

FIG. 8. (Color) Scanning electron microscope comparisons of stainless steel alloy 410 (top) and 304L (bottom). Pictures are cross sections through arc-damage locations. Several images were taken and these are representative of those images.

(psia)], 3.1 bar (47 psia), and 3.86 bar (58 psia). Weibull analysis of these data is given in Fig. 10. There is statistically no difference in switch performance between operation at 2.75 bar and 3.86 bar. The maximum allowable voltage is approximately $4.8 \mathrm{MV}$ for a $1 \%$ prefire rate for either pressure. It is evident that the threshold for increas-

TABLE III. Switch failure modes directly attributable to trigger electrode material. All data were for switches operated at 5.4 MV, peak current of $700 \mathrm{kA}$, with $1 \mathrm{C}$ total charge conducted per shot.

\begin{tabular}{lc}
\hline \hline Material (number of shots) & Failure mode \\
\hline CRES 304L (417) & High prefire rate $(2.5 \%)$ \\
Molybdenum (43) & Spacer flash, 40 shots \\
HD-17 (212) & Trigger flash, 45 shots \\
CW70E (100) & No failures \\
Mallory 1000 (100) & Optics life, 40 shots \\
\hline \hline
\end{tabular}




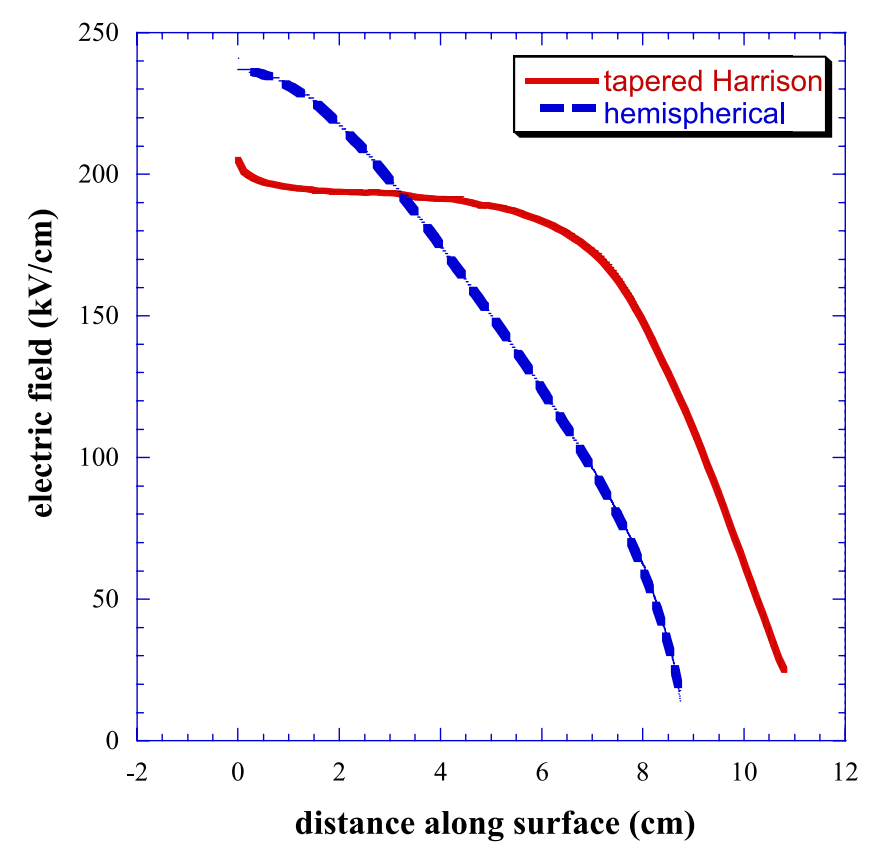

FIG. 9. (Color) Trigger electrode surface electric field for the modified-Harrison and hemispherical profiles. $d=0$ is at the axial center $(\rho=0)$ of the switch and $d$ increases along the surface with increasing $\rho$.

ing voltage hold-off by increasing $\mathrm{SF}_{6}$ pressure is exceeded at 2.75 bar for the hemispherical electrode design. Therefore, performance requirements for the refurbished $Z$ accelerator are not satisfied because the switch will not have a sufficient prefire rate at $5.4 \mathrm{MV}$, the voltage required to drive $21 \mathrm{MA}$ into the baseline load.

The electrode geometry was modified to de-enhance the electric field on axis $(\rho=0)$ and increase the area of uniform field between the electrodes as depicted in

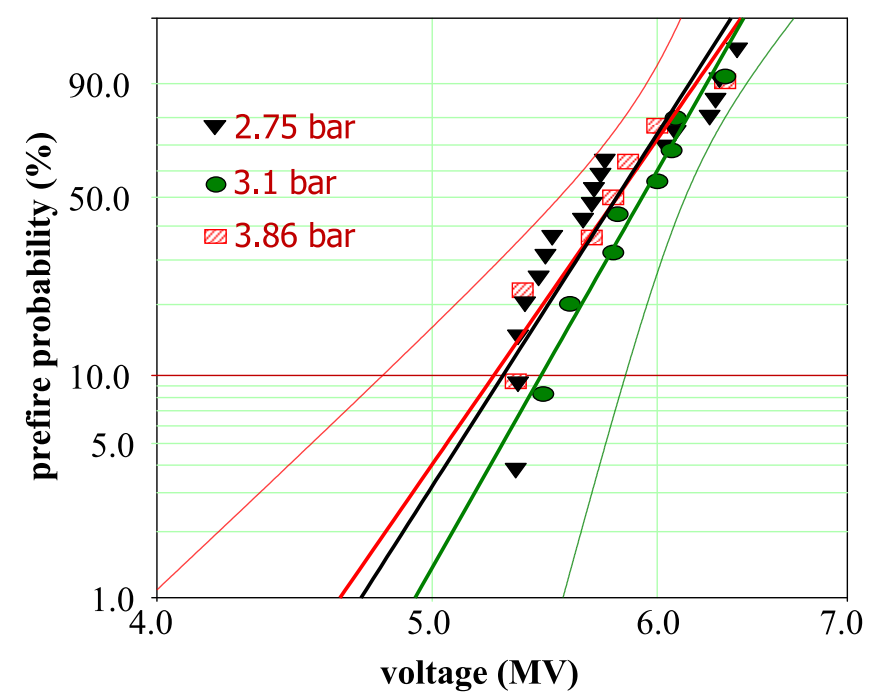

FIG. 10. (Color) Weibull analysis of self-closure data for hemispherical electrodes utilizing HD-17 trigger.
Fig. 9. This geometry addresses the major design flaw present in the hemispherical electrode by reducing the field on axis where current is conducted and electrode material is significantly eroded. The electrode profile is a modification to a uniform field profile developed for centimeter gaps by Harrison [45] that is a compromise between a Bruce and Rogowski profile. The profile was modified to preserve a uniform field in the geometry required.

Implementing this profile reduced the prefire probability significantly. Weibull analysis of self-closure experiments at $\mathrm{SF}_{6}$ pressures of 2.89 bar (44 psia), 3.17 bar (48 psia), and 3.44 bar (52 psia) for the modified-Harrison electrode profile with HD-17 electrodes is given in Fig. 11. A significant improvement is noted both in spread and in average breakdown voltage for increasing pressure by decreasing the ratio of peak field to average field. When utilizing HD-17 electrodes there is a $1 \%$ prefire rate for 2.89 bar at $6.8 \mathrm{MV}$ and for $3.44 \mathrm{bar}$ at $8.8 \mathrm{MV}$. At 5.4 MV the probability of a prefire is $0.001 \%$ for a $\mathrm{SF}_{6}$ pressure of 2.89 bar. In $433 Z_{20}$ shots there have been no trigger section prefires utilizing HD-17 with the modified-Harrison geometry.

Decreasing the peak field and improving the uniformity of the field in the trigger gap improved self-closure characteristics immensely. Comparison of stainless steel 304L electrode material with HD-17 electrode material utilizing the modified-Harrison electrode profile is given in Fig. 12. Self-closure data were normalized to the average breakdown voltage for each material (the average breakdown voltage is equated to a $50 \%$ prefire probability). A switch utilizing $304 \mathrm{~L}$ as the electrode material requires operation at a $23 \%$ lower voltage for the same prefire rate compared to a switch utilizing HD-17 as the electrode material. Three 100-shot experimental tests were conducted at 5.4 MV and

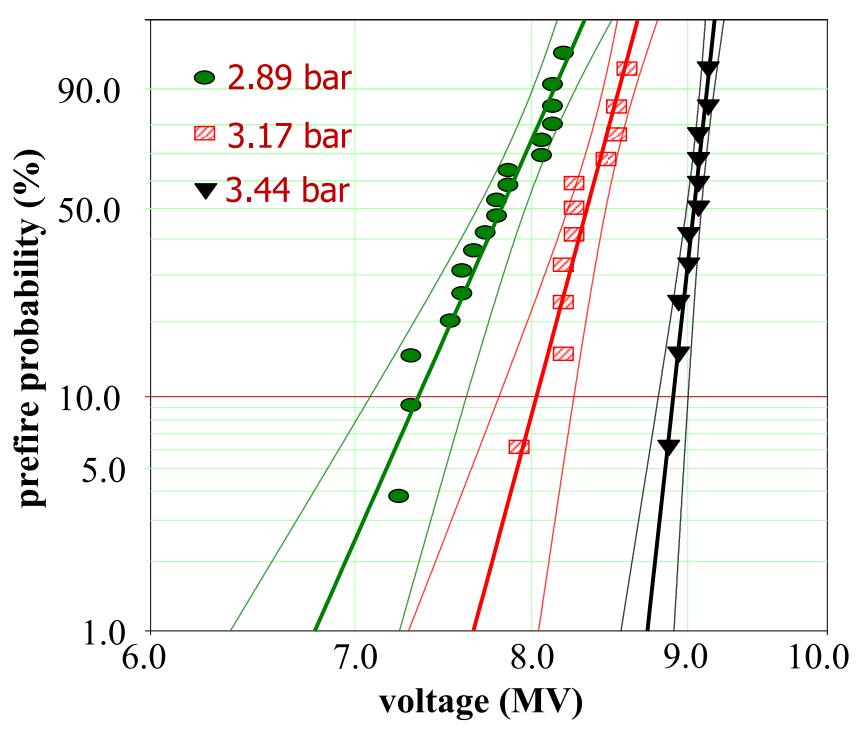

FIG. 11. (Color) Weibull analysis of self-closure data for the modified-Harrison profile utilizing HD-17 trigger electrodes. 


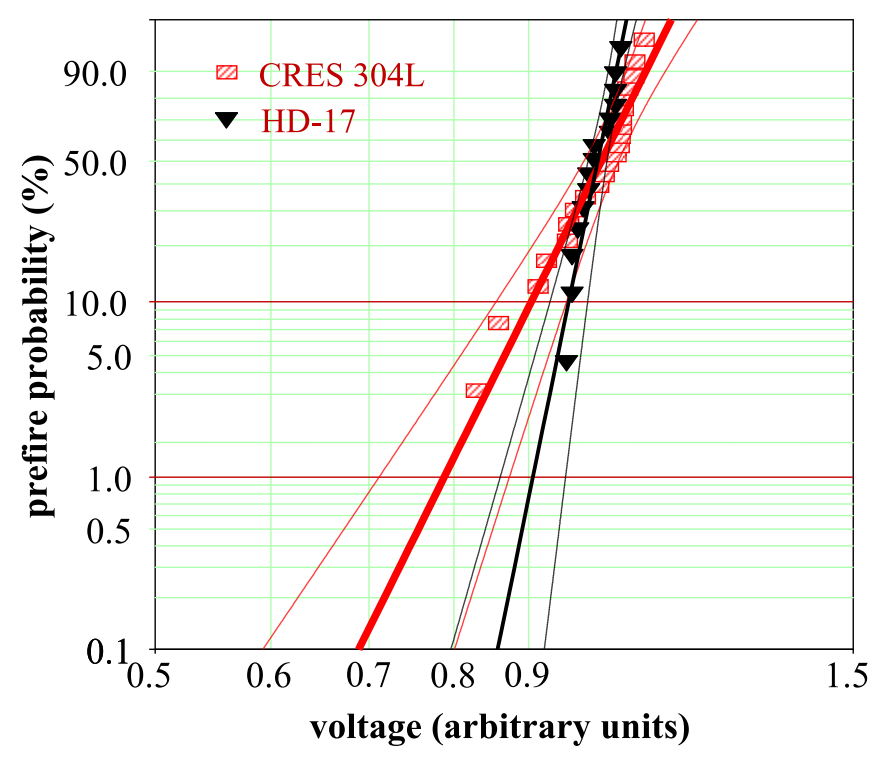

FIG. 12. (Color) Normalized prefire probability for CRES 304L and HD-17 trigger electrodes. Ninety-five percent confidence bands are also displayed.

2.89 bar with varying trigger gap distance, each of which resulted in a prefire rate of $\sim 2.5 \%$. This prefire rate is commensurate with the self-closure data depicted in Fig. 12.

Electrodes of similar refractory metal composition also exhibit a relatively small spread in self-closure voltage. A voltage spread of $\pm 4 \%$ is also observed in similar experiments utilizing Mallory $1000(90 \% \mathrm{~W}, 6 \% \mathrm{Ni}, 4 \% \mathrm{Cu}$, liquid sintered, manufactured by Mallory, Inc.), molybdenum, and $\mathrm{CW} 70 \mathrm{E}(68 \% \mathrm{~W}, 32 \% \mathrm{Cu}$, infiltrated, manufactured by Mi-Tech Metal) trigger electrodes.

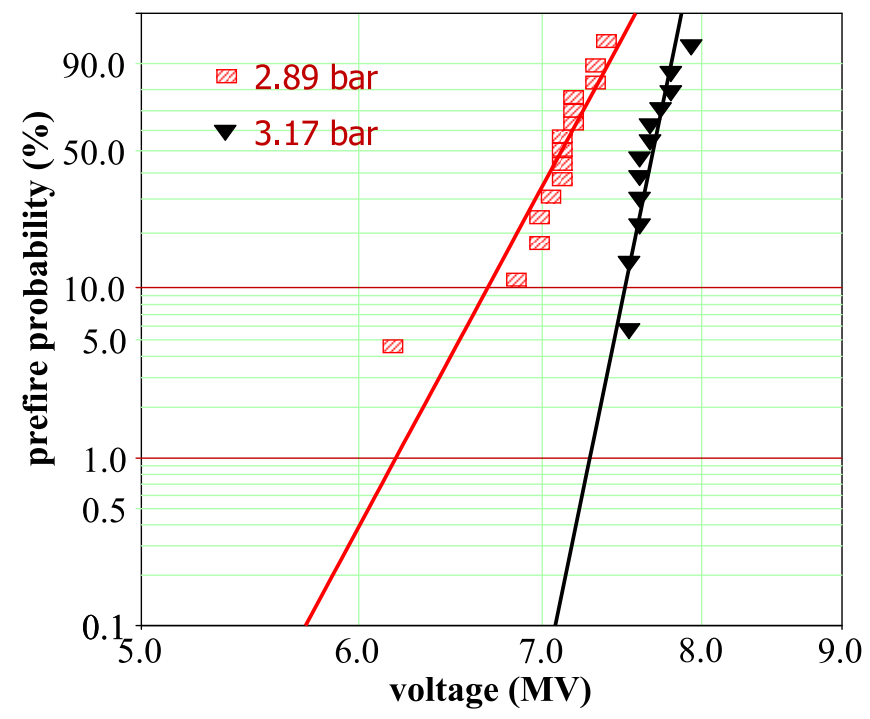

FIG. 13. (Color) Weibull analysis of self-closure data for the modified-Harrison profile utilizing CW70E trigger electrodes.
Weibull analysis of self-closure experiments at $\mathrm{SF}_{6}$ pressures of 2.89 bar (44 psia) and 3.17 bar (48 psia) for the modified-Harrison profile electrodes utilizing CW70E as the electrode material is given in Fig. 13. When utilizing this electrode configuration there is a $0.1 \%$ prefire rate for 2.89 bar at $5.75 \mathrm{MV}$ and for 3.17 bar at approximately $7 \mathrm{MV}$. The voltage spread of this configuration is similar to that of HD-17. In a $Z_{20}$ experimental run, there were no prefires and no other failures in 100 shots utilizing this configuration.

\section{Triggered performance}

Megavolt switching utilizing a laser beam as the triggering mechanism has been studied for several decades [15,46-55]. Focusing a laser beam into the center of a gap that is to be triggered can provide subnanosecond jitter while having the least probability of liberating electrode material due solely to laser ionization $[54,55]$. Utilization of $\sim 5 \mathrm{~ns}$ FWHM ultraviolet laser with wavelengths from 248 to $266 \mathrm{~nm}$ can provide nanosecond jitter, for centimeter length gaps in $\mathrm{SF}_{6}$, with only $\sim 2$ millijoules of laser energy [50] if the energy is focused properly. Subnanosecond jitter is possible when a large fraction of the gap $(\sim 25 \%)$ is ionized with focused laser energy [53,56].

The laser trigger system for the refurbished $Z$ accelerator consists of 36 independently controlled lasers, one for each switch. Each laser is capable of producing $35 \mathrm{~mJ}$ at $266 \mathrm{~nm}$ (frequency quadrupled $\mathrm{Nd}: Y A G$ ) with a $4 \mathrm{~ns}$ FWHM beam. The lasers, located in the basement of the facility, are directed into the switch through a series of optics as depicted in Fig. 14. From the basement the beam enters the optic housing and is reflected to a turning mirror to direct it into the trigger portion of the LTGS. The beam is focused into the switch utilizing a $50 \mathrm{~cm}$ focal length singlet that is coated with magnesium fluoride, an antire-

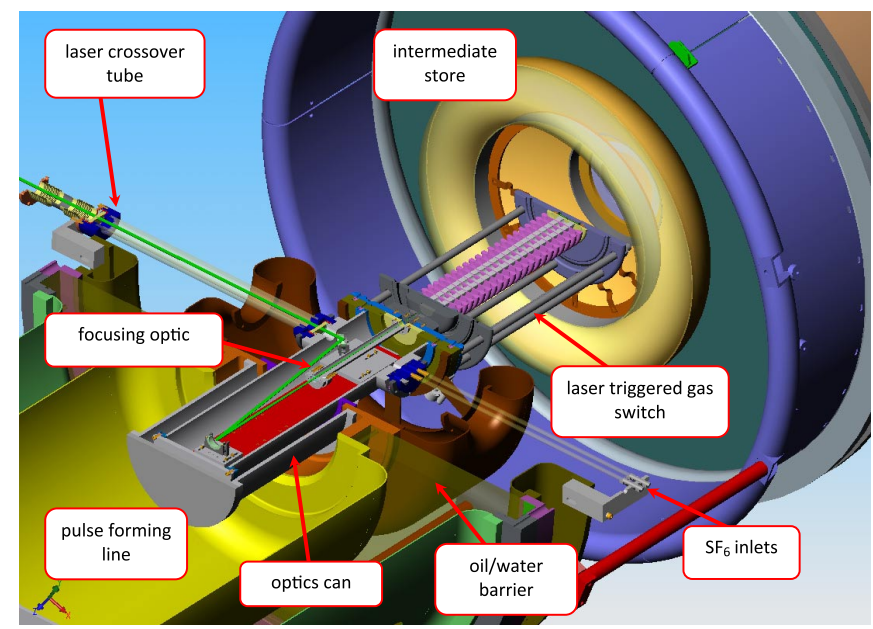

FIG. 14. (Color) The laser-trigger system for the refurbished $Z$ accelerator. 
flective. The total beam path has six mirrors and eight fused silica surfaces. There is a total of $43 \mathrm{~mm}$ of fused silica for the beam to pass through. The total optical path length is $11 \mathrm{~m}$. The total energy that arrives at the switch with pristine optics is $20 \mathrm{~mJ}$, or $\sim 60 \%$ of the energy at the output of the laser head.

The effectiveness of a focused laser to trigger the LTGS is dependent on the ratio of the effective length $l_{s}$ of sufficiently ionized gas by the focused laser energy to the total gap length $l_{g}$. This length is dependent on beam energy $E_{b}$, focal length $f$, and beam diameter $D$. The ratio of the focal length to the beam diameter is the $f_{\# \text {, where }}$

$$
f_{\text {\# }}=\frac{f}{D} .
$$

As the $f_{\#}$ increases the beam waist at the focal point will decrease, the energy density per unit length will decrease, and $l_{s}$ will increase for a Gaussian beam. The energy density sufficient to create a visible laser plasma utilizing a $248 \mathrm{~nm}$ beam in $\mathrm{SF}_{6}$ for pressures of interest is of order $2 \times 10^{10} \mathrm{~W} / \mathrm{cm}^{2}$ [48]. Therefore, there is a maximum $f_{\text {\# }}$ for a given electrode geometry to obtain a sufficiently ionized fraction of the gap to initiate closure.

Experiments were conducted by Clark and colleagues [56] to determine the dependence of visibly ionized $\mathrm{SF}_{6}$ plasma on the $f_{\#}$ of the optical system. The experimental setup consisted of two hemispherical electrodes with an $8.89 \mathrm{~cm}$ radius separated by a $4.6 \mathrm{~cm}$ gap with no applied voltage. F-numbers of $41.67,62.5$, and 83.33 were selected to determine the dependence of visible spark length on $f_{\# \text {. }}$. Figure 15 depicts the visible spark length $l_{v}$ versus output

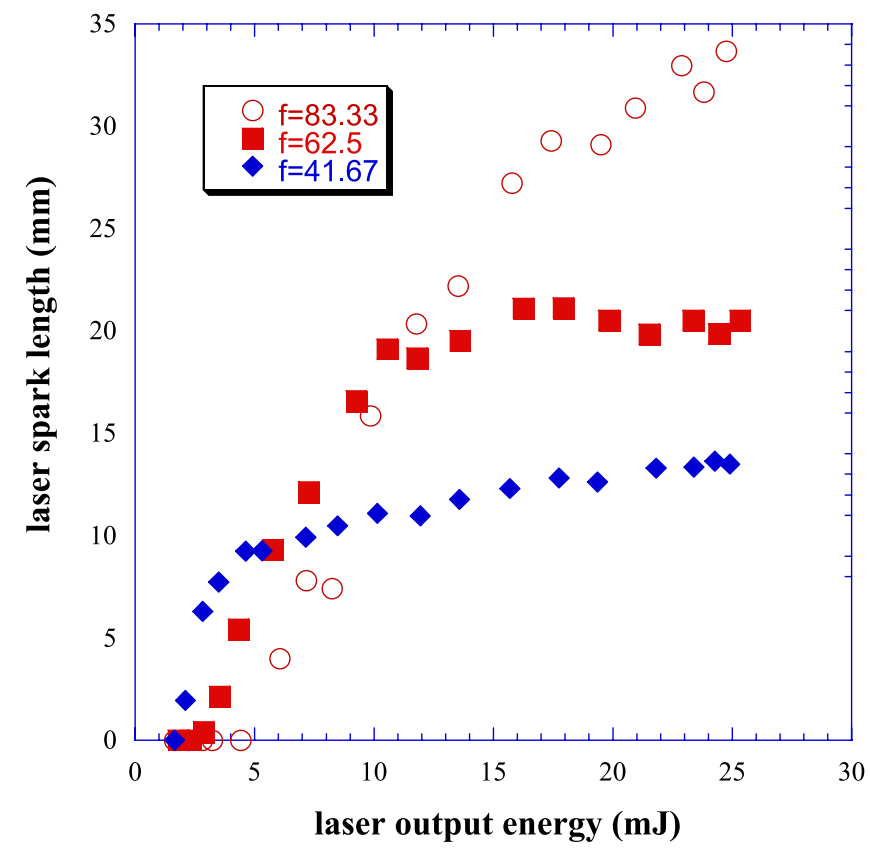

FIG. 15. (Color) Laser energy versus visible spark length for three $f$-numbers. laser energy for selected $f_{\#}$. The visible spark length was recorded by a thermoelectrically cooled CCD camera model ST-8XMEI manufactured by SBIG Astronomical Instruments. It was shown by Savage and colleagues that the effective electrical length of the spark $l_{s}$ is proportional to $l_{v}$ [57]. The minimum energy required to produce a

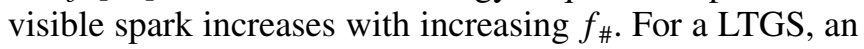
$f_{\text {\# }}$ that is high will result in a system that is sensitive to optics degradation. An $f_{\#}$ that is low will result in an insufficiently ionized ratio of the gap to be triggered. Therefore, the desired optics lifetime and ability to close the gap dictate an optimum $f_{\#}$. An $f_{\#}$ of 41.67 was selected for the LTGS discussed in the manuscript to provide a sufficient ionized length for the desired closure time of the gap and to meet requirements for switch lifetime ( $>150$ shots without maintenance).

Increasing laser energy will increase $l_{v}$ varying in length from 0 millimeters at 0 millijoules, to 12 millimeters at 20 millijoules. The fraction of the gap that is electrically shorted by the ionized channel determines the minimum $f_{\text {\# }}$ that is required to trigger the gap with a given jitter. This fraction is defined as

$$
R_{g}=\frac{l_{v}}{l_{g}}
$$

Figure 16 depicts the relationship between $R_{g}$ and switch jitter. At least $30 \%$ of the gap must be shorted by visible laser plasma for subnanosecond jitter. The electrical length of the spark is approximately twice that of the visible spark length [56]. Therefore, the visible spark length is a con-

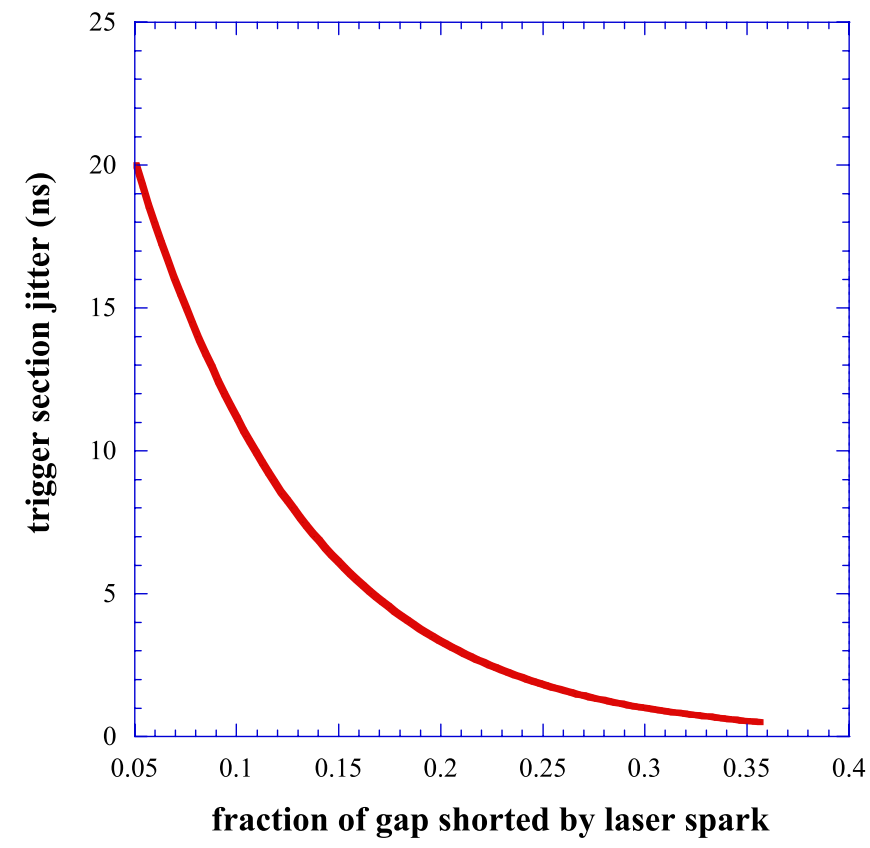

FIG. 16. (Color) Trigger section jitter as a function of the fraction of the trigger gap shorted by the visible laser spark. 


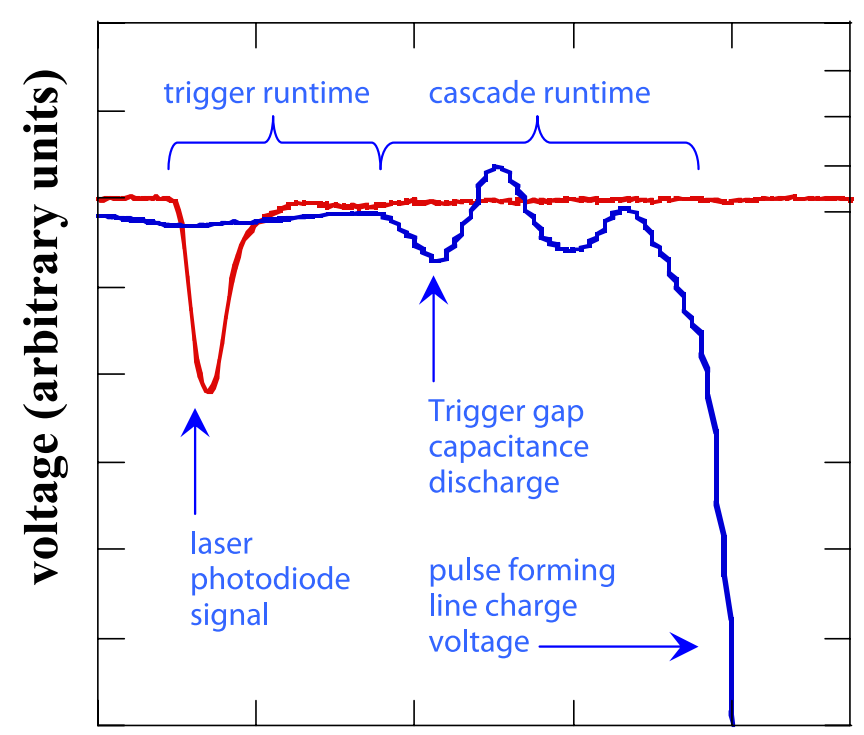

\section{time (arbitrary units)}

FIG. 17. (Color) Example voltage traces from the diagnostics utilized to determine trigger and cascade runtimes.

servative indication of sufficient laser energy to close a gap with a given jitter.

The operation of each switch section is diagnosed via a D-dot monitor located in the pulse-forming line adjacent to the trigger section. It has adequate sensitivity to detect a $\sim 20 \mathrm{~J}$ capacitive discharge from the trigger gap $(60 \mathrm{pF}$ capacitor at $800 \mathrm{kV}$ ) when the focused laser beam initially ionizes a portion of the trigger gap. The time between laser arrival at the gap and this capacitive discharge is defined as trigger runtime. The time between trigger gap discharge and conduction of current as detected by a downstream monitor is defined as the cascade runtime. An example of trigger and cascade runtime is given in Fig. 17. The relative accuracy of this monitor is $\pm 1 \mathrm{~ns}$ as confirmed by streak and framing camera images with respect to a known zero time marker.

Figure 18 depicts trigger runtime as a function of laser energy $E_{l}$ utilizing CW70E electrodes. The curve fit in Fig. 18 indicates trigger runtime $\tau_{t}$ is

$$
\tau_{t} \propto E_{l}^{-0.43} \approx \frac{1}{\sqrt{E_{l}}} .
$$

Experiments were conducted utilizing an $f_{\#}$ of 41.67 to determine the minimum laser energy required to trigger the switch. Laser energy was varied from 20 millijoules to $\sim 1$ millijoule, with successful triggering at $1 \mathrm{~mJ}$ as depicted in Fig. 18. Analyzing the trigger jitter $\sigma_{t}$ from Fig. 18 we find

$$
\sigma_{t} \propto E_{l}^{-0.96} \approx \frac{1}{E_{l}} .
$$

Assuming a power fit to the increase in trigger jitter after

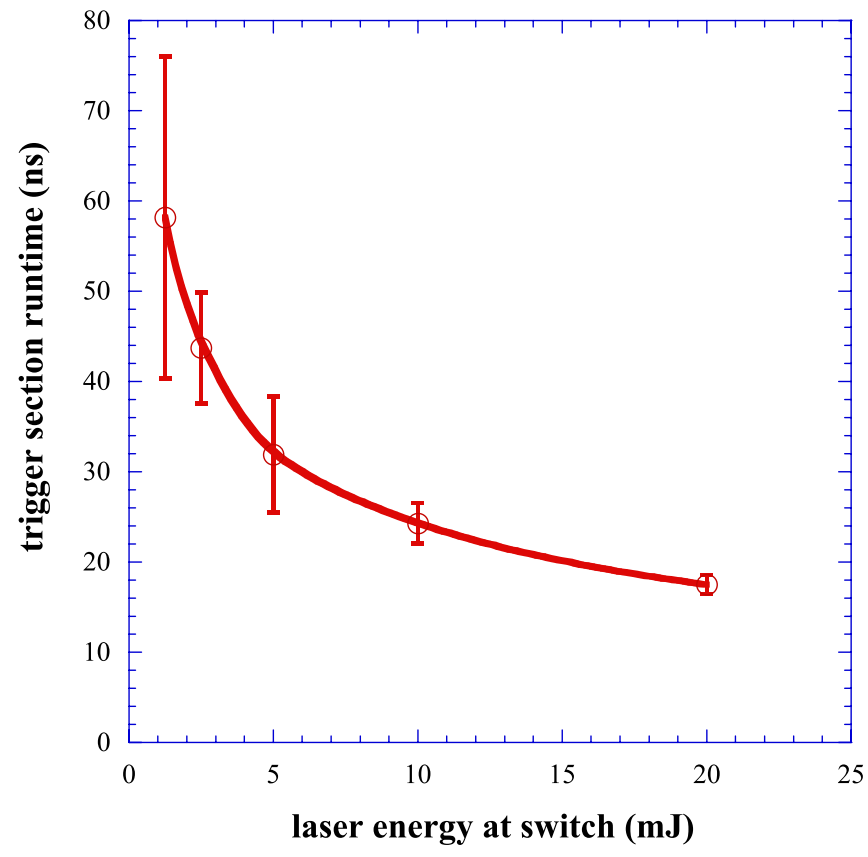

FIG. 18. (Color) Average trigger runtime as a function of laser energy. The laser beam is focused at the center of a $4.6 \mathrm{~cm}$ gap with a $50 \mathrm{~cm}$ focal length lens. Vertical bars represent a 1- $\sigma$ timing jitter of ten-shot sets.

100 shots, the trigger jitter will be less than $4 \mathrm{~ns}$ after $\sim 800$ shots in this configuration. Similar optic performance was noted with HD-17, CW70E, and stainless steel 304L trigger electrodes. When utilizing Mallory 1000 and molybdenum trigger electrodes the trigger runtime was worsened

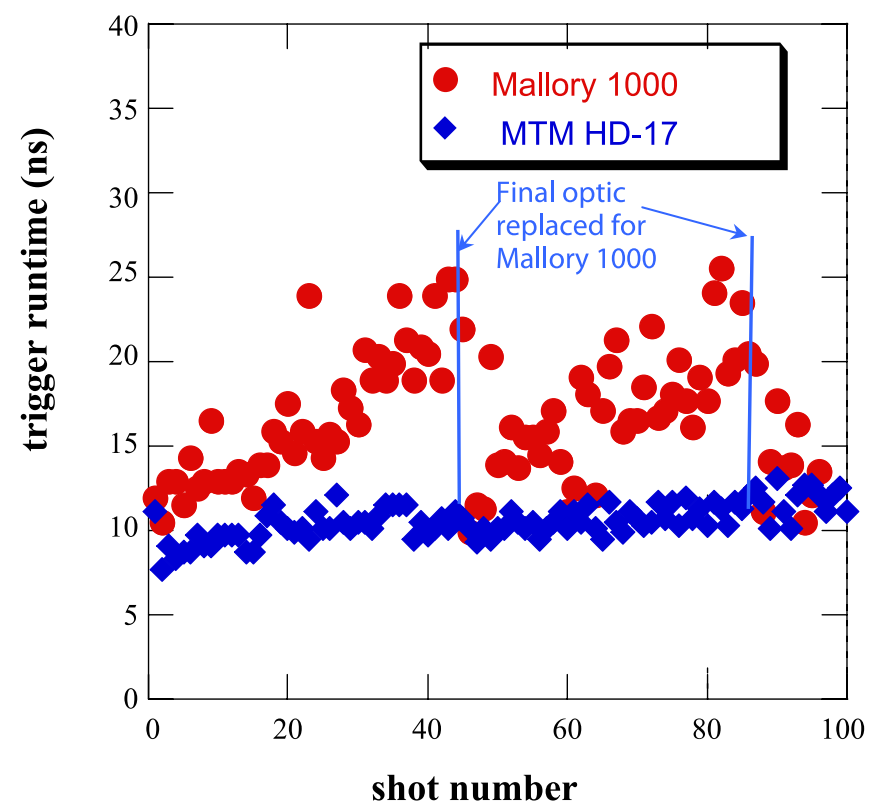

FIG. 19. (Color) Comparison of trigger runtime for Mallory 1000 and HD-17, both are $90 \% \mathrm{~W}, 10 \% \mathrm{Cu}-\mathrm{Ni}$ sintered materials. 
TABLE IV. Trigger runtime and jitter as a function of trigger electrode material.

\begin{tabular}{lccc}
\hline \hline $\begin{array}{l}\text { Material (number } \\
\text { of shots) }\end{array}$ & $\begin{array}{c}\text { Runtime } \\
\text { (ns) }\end{array}$ & $\begin{array}{c}\text { Jitter } \\
\text { (ns) }\end{array}$ & Failure mode \\
\hline CRES 304L (417) & 11.4 & 2.4 & High prefire rate $(2.5 \%)$ \\
Molybdenum (43) & 13.1 & 2.1 & Spacer flash, 40 shots \\
HD-17 (212) & 11 & 1 & Trigger flash, 45 shots \\
CW70E (100) & 10.3 & 1 & No failures \\
Mallory 1000 (100) & 16.8 & 4 & Optics life, 50 shots \\
\hline \hline
\end{tabular}

substantially having only a $\sim 40$ shot optics life as depicted in Fig. 19. A summary of trigger runtime and jitter performance for each tested electrode material is given in Table IV.

\section{Cascade section design and performance}

The self-closing cascade section has 22 individual gaps of varying length. Gap distances were tailored in length to ensure a uniform axial electric field from gap to gap, a criterion deemed necessary for the first redesign of gas switches for PBFA II [58]. The peak field for this LTGS is $205 \pm 6 \mathrm{kV} / \mathrm{cm}$ for $6 \mathrm{MV}$ operation as depicted in Fig. 20. Reducing the peak field from $220 \mathrm{kV} / \mathrm{cm}$ to $205 \mathrm{kV} / \mathrm{cm}$ in addition to reducing the nonuniformity from $\pm 10 \%$ to $\pm 3 \%$ decreased the self-closure voltage spread by a factor of 2 as depicted in Fig. 21.

The operating pressure $p$ and the electric field overstress $E_{c}$ applied to the cascade section by closing the trigger gap affect the cascade section runtime and jitter. The magni-

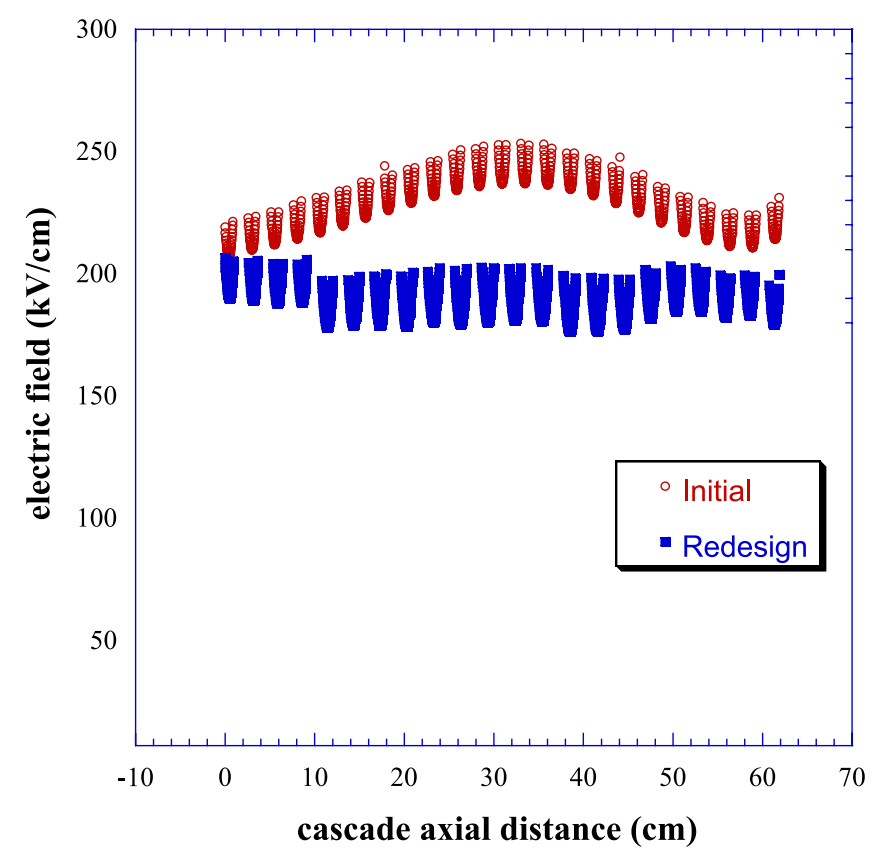

FIG. 20. (Color) Cascade electric field in each gap at $6 \mathrm{MV}$ charge. The trigger gap is at $d=0$.

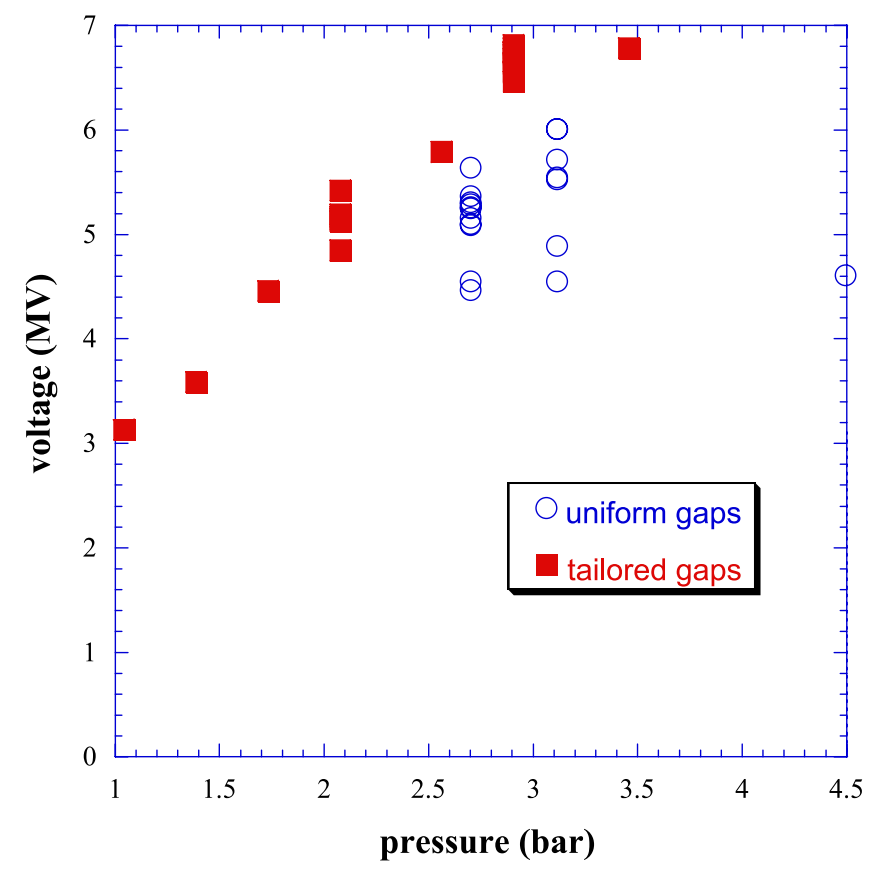

FIG. 21. (Color) Cascade self-closure voltage versus pressure. The initial design utilized uniform gaps and the redesign, tailored lengths to achieve uniform and lower peak field.

tude and distribution of the overvoltage wave from the trigger gap is dependent on the geometry of the switch and the surrounding structure. When the trigger gap closes, there is an increase in peak electric field of $56 \%$ from $205 \mathrm{kV} / \mathrm{cm}$ to $320 \mathrm{kV} / \mathrm{cm}$ for a $6 \mathrm{MV}$ charge in the adjacent cascade gap (the gap at $2 \mathrm{~cm}$ in Fig. 20). Figure 22 depicts the cascade section runtime as a function of reduced field $E_{c} / p$ for $\sim 700$ shots on 15 LTGS switch builds. For any single switch build the jitter is less than $6 \mathrm{~ns}$ if

$$
\frac{E_{c}}{p}>96 \frac{\mathrm{kV}}{\mathrm{cm} \cdot \mathrm{bar}} .
$$

Not all switch builds have identical average runtime, but all builds were within $5 \mathrm{~ns}$ of each others average.

A similar parameter was determined for sufficiently low jitter operation for the PBFA II gas switch [59]. For an acceptable PBFA II prefire rate,

$$
\frac{E_{t}}{p}>45 \frac{\mathrm{kV}}{\mathrm{cm} \cdot \mathrm{bar}},
$$

where $E_{t}$ is the lowest electric field in the trigger gap, occurring in the center of the gap. Convert $E_{t} / p$ to an equivalent $E_{c} / p$ value, the PBFA II switch was deemed to have low jitter when

$$
\frac{E}{p}>110 \frac{\mathrm{kV}}{\mathrm{cm} \cdot \mathrm{bar}}
$$

for $\sim 5 \mathrm{MV}$ operation. This is consistent with performance of the LTGS for the refurbished $Z$ accelerator at 


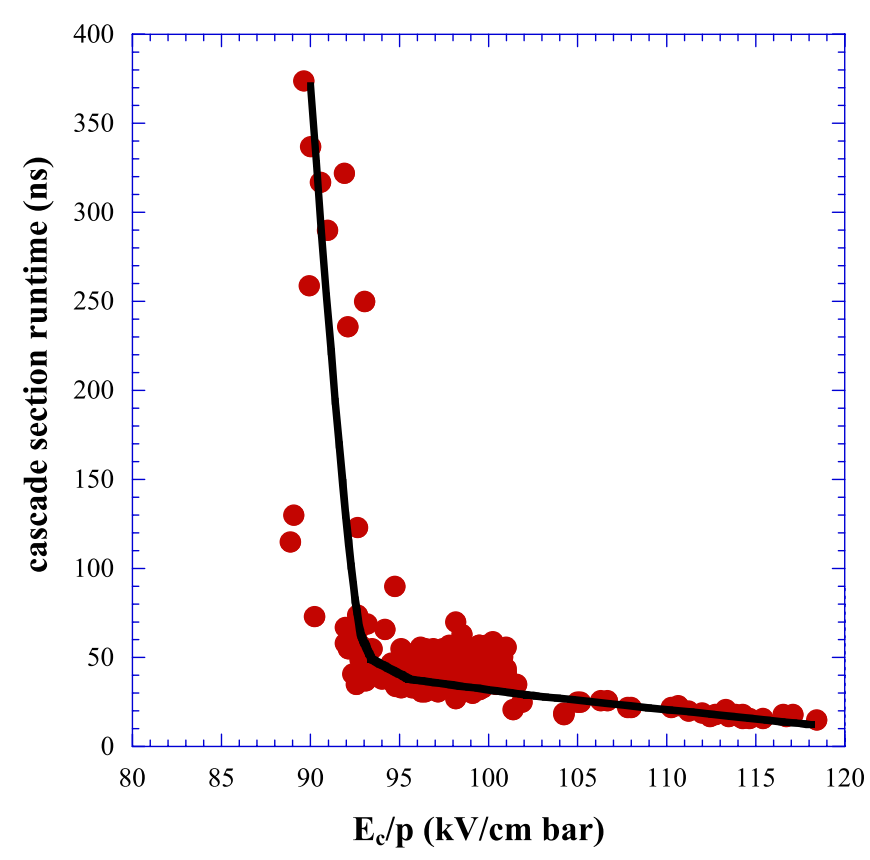

FIG. 22. (Color) Cascade section runtime as a function of the electric field $E_{c}$ in the adjacent cascade gap to the trigger gap, and the operating pressure $p$. Data are for 700 shots on the redesigned LTGS.

$110 \mathrm{kV} / \mathrm{cm}$-bar and exceeds the requirement defined in Eq. (7) to meet present performance goals.

Streak camera data show that the higher the overstress of the adjacent gaps to the trigger section the lower the cascade jitter. Figure 23 depicts an average of four shots each for 2.75 bar (42 psia) and 3.03 bar (46 psia) operation. The voltage for the 42 psia shots was $\sim 5.25 \mathrm{MV}$ and $\sim 5.35 \mathrm{MV}$ for the 46 psia shots. Gap 0 signifies the time at which the trigger gap capacitive discharge is detected. Electrical closure of the trigger gap is detected approximately $12 \mathrm{~ns}$ after the laser arrives in the switch and approximately $10 \mathrm{~ns}$ after first light is detected by a photodiode mounted near the switch. The time between gap 0 closure and gap 1 closure is the runtime of the first gap. The runtime of first cascade gap is approximately $50 \%$ of the total cascade section runtime (the time from gap 0 to gap 22 ) and also accounts for approximately $50 \%$ of the total cascade section jitter. The cascade section demonstrated a total jitter of 4.2 ns for three independent 100-shot experimental tests at $5.4 \pm 0.04 \mathrm{MV}$ for a pressure of $2.89 \mathrm{bar}$ (44 psia) corresponding to $20 \pm 0.15 \mathrm{MA}$ in $100 \mathrm{~ns}$ into the baseline z-pinch load. Increasing $E_{c} / p$ is required to reduce switch jitter further and designs that achieve this are presently being investigated in ongoing switch testing.

\section{PREPARATION PROTOCOLS FOR ELECTRODES AND INSULATORS}

In August 2005, frequent random flashing of insulators at any operating level precluded the possibility of deliver-

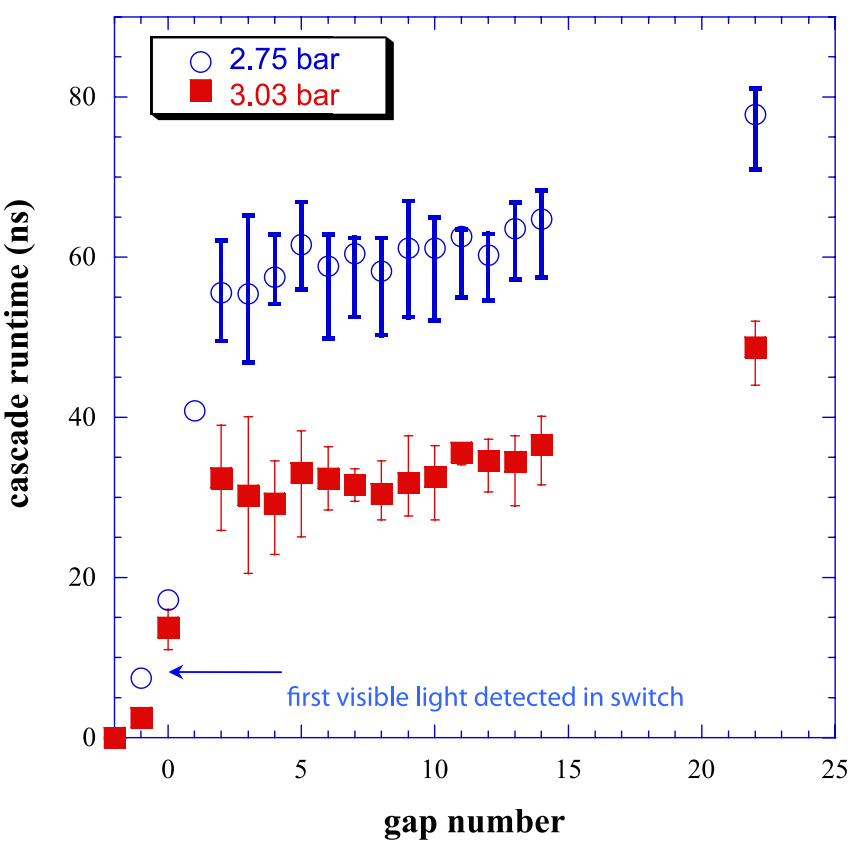

FIG. 23. (Color) Cascade breakdown times for four shots averaged each at $42 \mathrm{psia}$ and $46 \mathrm{psia}$. The voltage for the $42 \mathrm{psia}$ shots was $\sim 5.25 \mathrm{MV}$ and $\sim 5.35 \mathrm{MV}$ for the 46 psia shots. Gap 0 signifies the time at which the trigger gap capacitive discharge is detected. The time between gap 0 closure and gap 1 is the runtime of the first gap. The error bars represent the spread in the data for those four shots. The first 14 gaps nearest the trigger section are visible, the others are obstructed from the field of view.

ing a suitable gas switch for the refurbished $Z$ accelerator. There were an average of ten shots between either trigger or cascade section flashes. New protocols were instituted that dramatically reduced flashing. They are (i) controlled cleaning and handling procedures for insulators, (ii) controlled fabrication and preparation procedures for electrodes, (iii) immediate and turbulent gas purging after a shot, and (iv) electrically shielding all triple points. The purpose of these procedures is to reduce infant mortality and random flashovers.

The cascade section insulators are polymethyl methacrylate (PMMA, or acrylic), ultraviolet transparent, with no blue dye. The cleaning procedure developed is outlined in Appendix A 1 with all handling protocols following ISO 8 (class 100,000). Implementing this procedure decreased the probability of flashover more than an order of magnitude to less than $1 \%$ at $5.4 \mathrm{MV}$ at 2.89 bar (44 psia). Cascade housings have survived peak switch voltages of $6.9 \mathrm{MV}$ at 3.44 bar and trigger insulators up to the equivalent of $9 \mathrm{MV}$ on the gas switch at 3.44 bar.

Electrode handling procedures are implemented to mitigate the possibility of random failures caused by contamination and inconsistencies in part fabrication. The preparation procedure developed for stainless steel $304 \mathrm{~L}$ and 410 is outlined in Appendix A 2 with all handling 


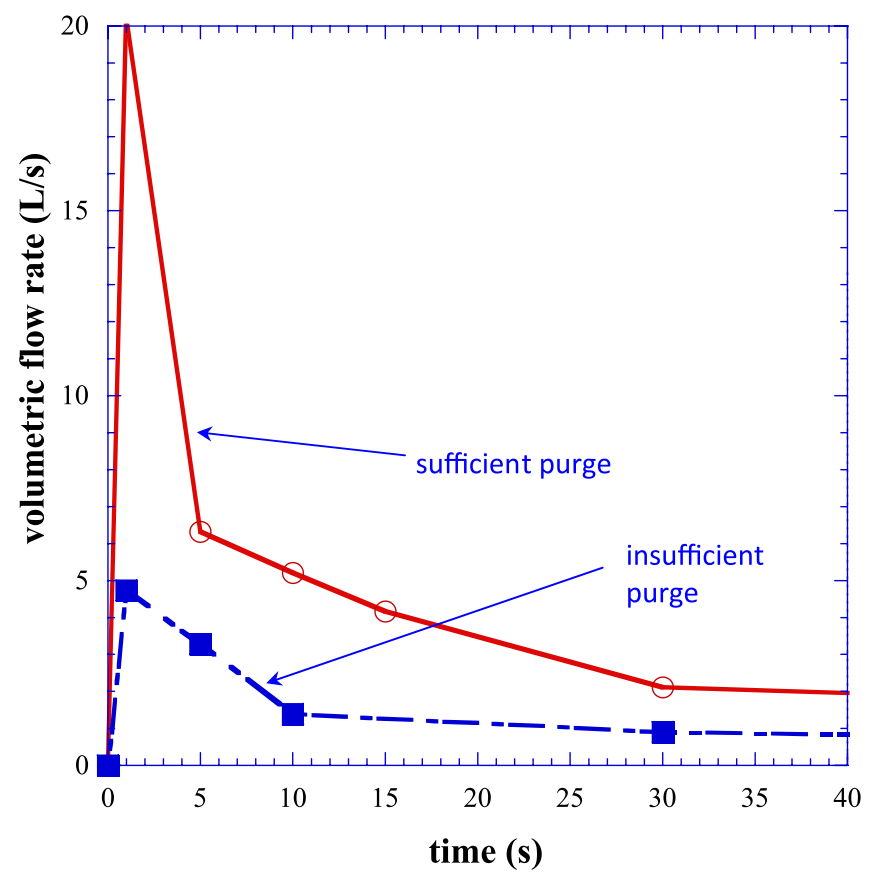

FIG. 24. (Color) $\mathrm{SF}_{6}$ purge after a shot. The switch volume is $60 \mathrm{~L}$.

protocols following ISO 8 (class 100,000). The preparation procedure developed for refractory based metals is outlined in Appendix A 3 with all handling protocols following ISO 8 (class 100,000).

Sulfur-hexafluoride is purged from the switch immediately following a discharge in order to mitigate insulator and optic contamination as a result of liberated electrode material in the form of $\mathrm{SF}_{6}$ by-products. The switch is exhausted into a low-pressure ( $<1$ psia) volume $50 \%$ the size of the switch immediately after the shot. A fourfold decrease in catastrophic flashover was achieved by increasing the peak flow rate as depicted in Fig. 24 from $5 \mathrm{~L} / \mathrm{s}$ to $20 \mathrm{~L} / \mathrm{s}$.

\section{SUMMARY AND FUTURE WORK}

The laser triggered gas switch developed for the refurbished $Z$ accelerator operates at $5.4 \mathrm{MV}, 700 \mathrm{kA}$, using sulfur-hexafluoride as the fill gas. At this operating level, switch jitter is $5 \mathrm{~ns}$, the prefire rate is less than $0.1 \%$, the average optic lifetime is greater than 200 shots, the switch lifetime is $\sim 110 \pm 10$ shots, and the flashover rate is less than $1 \%$. Low-erosion electrode material, modifications to internal switch geometry, advances in material processing, and testing methodology have greatly improved our understanding of multimegavolt laser triggered gas switches. The switch presented in this manuscript was extensively tested at the $5.4 \mathrm{MV}$ level and is presently being tested at the 6.1 MV level to determine reliability at a higher operating level. We are pursuing new switch designs to provide a lower jitter switch with increased lifetime at the $6.25 \mathrm{MV}$, 800 MA level.

\section{ACKNOWLEDGMENTS}

The authors would like to thank Keegan Shelton from MPCL for his assistance with the preparation and cleaning of electrodes. The authors would like to thank Dick Grant from Sandia National Labs for his assistance with materials analysis.

\section{APPENDIX A}

Cleaning, fabricating, and handling protocols were implemented for insulators and electrodes to reduce the variability between switch assemblies. Each procedure follows ISO 8 (class 100000 ) clean room protocols. They are each described below.

\section{Acrylic insulator cleaning procedure: Follows ISO 8 (class 100,000) handling protocols}

(i) Wash using warm water and Simple Green with a lintfree cloth; (ii) wipe dry with a high-grade lint-free cloth; (iii) wipe in the circumferential direction with hexane and a high-grade lint-free cloth; (iv) blow housing with charge neutralized nitrogen at 1.37 bar (22 psia); (v) store in sealed plastic bags.

\section{Stainless steel electrode preparation procedure: Follows ISO 8 (class 100,000) handling protocols}

(i) Bead blast with clean glass bead; (ii) passivate per ASTM spec A967 (a Nitric 2 bath, 20 to 45 volume percent of nitric acid at room temperature for at least 30 minutes); (iii) evacuate furnace to high vacuum for 20 minutes; (iv) heat to $200 \mathrm{C}$, increasing oven $10^{\circ}$ per minute for $20 \mathrm{mi}$ nutes; (v) heat to $600 \mathrm{C}$, increasing oven $20^{\circ}$ per minute for 20 minutes; (vi) heat to $800 \mathrm{C}$, increasing oven $10^{\circ}$ per minute for 20 minutes; (vii) purge furnace to inert environment (argon); (viii) hold $800 \mathrm{C}$ and displace argon with hydrogen; (ix) heat to $1100 \mathrm{C}$, increasing oven $20^{\circ}$ per minute for 10 minutes; (x) hydrogen fire at $1100 \mathrm{C}$ for 45 minutes; (xi) cool to $850 \mathrm{C}$ and begin argon purge for $1 \mathrm{~min}$; (xii) purge with argon for 85 minutes; (xiii) vacuum fire at $850 \mathrm{C}$ for 120 minutes; (xiv) cool to $825 \mathrm{C}$ for $1 \mathrm{~min}$; (xv) hold at $825 \mathrm{C}$ for 10 minutes; (xvi) cool to $800 \mathrm{C}$ for $1 \mathrm{~min}$; (xvii) vacuum fire at $800 \mathrm{C}$ for 360 minutes $\left(10^{-5}-10^{-6}\right.$ torr); (xviii) backfill with argon allowing furnace to cool to $200 \mathrm{C}$; (xix) backfill with argon until room temperature is reached; $(\mathrm{xx})$ wrap in high-grade aluminum foil for storage.

\section{Refractory metal electrode handling procedure: Follows ISO 8 (class 100,000) handling protocols}

(i) Evacuate chamber to $10^{-5}$ torr; (ii) increase to $800 \mathrm{C}$ in 30 minutes; (iii) bake at $800 \mathrm{C}$ for 60 minutes; (iv) cool to room temperature; (v) backfill with argon to atmosphere once part is cool. 
[1] W. A. Stygar et al., Phys. Rev. ST Accel. Beams 10, 030401 (2007).

[2] J. R. Woodworth et al., IEEE Trans. Plasma Sci. 33, 2051 (2005).

[3] D. L. Johnson, in Proceedings of the 1st IEEE International Pulsed Conference, Lubbock, 1976, edited by T. Burkes and M. Kristiansen (IEEE, Piscataway, NJ, 1976), p. IE2.

[4] L. J. Demeter, G. Frazier, and T. Naff, IEEE Trans. Nucl. Sci. 28, 2931 (1981).

[5] T.H. Martin et al., IEEE Trans. Nucl. Sci. 28, 3365 (1981).

[6] J. J. Ramirez et al., in Proceedings of 6th IEEE International Pulsed Power Conference, Arlington, 1987, edited by P. Turchi and B. Bernstein (IEEE, Piscataway, NJ, 1987), p. 294.

[7] D. D. Bloomquist et al., in Proceedings of 6th IEEE International Pulsed Power Conference, Arlington, 1987, Ref. [6], p. 310.

[8] D. R. Humphreys et al., in Proceedings of 5th IEEE International Pulsed Power Conference, Arlington, 1985, edited by M. Rose and P. Turchi (IEEE, Piscataway, NJ, 1985), p. 262.

[9] R. B. Spielman et al., in 11th IEEE International Pulsed Power Conference, Baltimore, 1997, edited by G. Cooperstein and I. Vitkovitsky (IEEE, Piscataway, NJ, 1997), p. 709.

[10] M.E. Savage et al., in 16th IEEE Pulsed Power Conference, Albuquerque, NM, 2007, edited by E. Schamiloglu and F. Peterkin (IEEE, Piscataway, NJ, 2007), p. 979.

[11] B. H. Lee, J. Korean Phys. Soc. 34, 248 (1999).

[12] S. N. Volkov et al., in 12th IEEE International Pulsed Power Conference, Monterey, 1999, edited by C. Stalling and H. Kirbie (IEEE, Piscataway, NJ, 1999), p. 1179.

[13] G. J. Denison et al., in Proceedings of 6th IEEE International Pulsed Power Conference, Arlington, 1987, Ref. [6], p. 490.

[14] G. J. Denison et al., in 7th IEEE International Pulsed Power Conference, Monterey, 1989, edited by B. Bernstein and J. Shannon, p. 579.

[15] J. R. Woodworth, R. G. Adams, and C. A. Frost, IEEE Trans. Plasma Sci. 10, 257 (1982).

[16] D. D. Bloomquist et al., in Proceedings of 5th IEEE International Pulsed Power Conference, Arlington, 1985, Ref. [8], p. 266.

[17] J. W. Douglas, W. F. J. Crewson, and C. H. Jones, Defense Nuclear Agency Report No. 001-79-C-0269, Washington D.C., Final report December 1979.

[18] R. G. Adams et al., in 4th IEEE International Pulsed Power Conference, Albuquerque, 1983 (IEEE, Piscataway, NJ, 1983), edited by T. Martin and M. Rose, p. 613.

[19] H. An et al., Plasma Sci. Technol. 8, 602 (2006).

[20] B. N. Turman et al., in 4th IEEE International Pulsed Power Conference, Albuquerque, 1983, Ref. [18], p. 617.

[21] J.P. Corley et al., in 13th IEEE International Pulsed Power Conference, Las Vegas, 2001, edited by R. Reinovsky and M. Newton (IEEE, Piscataway, NJ, 2001), p. 1778.
[22] J.P. Corley et al., in 14th IEEE International Pulsed Power Conference, Dallas, 2003, edited by M. Giesselmann and A. Neuber (IEEE, Piscataway, NJ, 2003), p. 875.

[23] M. A. Kemp et al., IEEE Trans. Plasma Sci. 33, 1245 (2005).

[24] M. A. Kemp, R. D. Curry, and S.D. Kovaleski, IEEE Trans. Plasma Sci. 34, 95 (2006).

[25] D. L. Johnson, J. P. VanDevender, and T. H. Martin, IEEE Trans. Plasma Sci. PS-8, 204 (1980).

[26] J. R. Woodworth et al., IEEE Trans. Plasma Sci. 32, 1778 (2004).

[27] W. A. Stygar et al, in 12th IEEE International Pulsed Power Conference, Monterey, 1999, Ref. [12], p. 454.

[28] D. J. Anderson and D. Briand, in 14th IEEE International Pulsed Power Conference, Dallas, 2003, Ref. [22], p. 183.

[29] P. K. Rambo et al., Appl. Opt. 44, 2421 (2005).

[30] K. W. Struve, H. C. Harjes, and J. P. Corley, in 16th IEEE Pulsed Power Conference, Albuquerque, NM, 2007, Ref. [10], p. 985.

[31] J.R. Woodworth et al., in 15th IEEE Pulsed Power Conference, Monterey, CA, 2005, p. 639.

[32] B. N. Turman and D. R. Humphreys, in 7th IEEE International Pulsed Power Conference, Monterey, 1989, Ref. [14], p. 555.

[33] B. N. Turman, A. E. Rodriguez, and K. J. Touryan, in 8th IEEE International Pulsed Power Conference, San Diego, 1991, edited by R. White and K. Prestwich, p. 319.

[34] S. E. Rosenthal et al., in 14th IEEE International Pulsed Power Conference, Dallas, 2003, Ref. [22], p. 163.

[35] K. R. LeChien and J. M. Gahl, IEEE Trans. Plasma Sci. 34, 1646 (2006).

[36] V. E. Ilin and S. V. Lebedev, Sov. Phys. Tech. Phys. 7, 717 (1963).

[37] A.L. Donaldson and M. Kristiansen, in 7th IEEE International Pulsed Power Conference, Monterey, 1989, Ref. [14], p. 83.

[38] S. O. Shiryaeva and A. I. Grigorev, Tech. Phys. Lett. 19, 443 (1993).

[39] A. L. Donaldson et al., J. Appl. Phys. 57, 4981 (1985).

[40] A.L. Donaldson et al., IEEE Trans. Magn. 22, 1441 (1986).

[41] A. L. Donaldson and M. Kristiansen, Report No. AAIA89-2515.

[42] A. L. Donaldson, T. G. Engel, and M. Kristiansen, IEEE Trans. Magn. 25, 138 (1989).

[43] J. M. Koutsoubis and S. J. MacGregor, J. Phys. D 33, 1093 (2000).

[44] A. L. Donaldson, F. M. Lehr, and M. Kristiansen, in Space Structures, Power, and Power Conditioning (SPIE, Bellingham, WA, 1988), p. 273.

[45] J. A. Harrison, Br. J. Appl. Phys. 18, 1617 (1967).

[46] A.H. Guenther and J.R. Bettis, IEEE J. Quantum Electron. 3, 581 (1967).

[47] A. H. Guenther and J.R. Bettis, J. Phys. D 11, 1577 (1978).

[48] W. R. Rapoport, J. Goldhar, and J. R. Murray, IEEE Trans. Plasma Sci. PS-8, 167 (1980).

[49] R. A. Dougal and P.F. Williams, J. Phys. D 17, 903 (1984).

[50] J. R. Woodworth et al., J. Appl. Phys. 56, 1382 (1984). 
[51] C. B. Edwards, F. O’Neill, and M. J. Shaw, J. Phys. E 18, 136 (1985).

[52] H. Kusama and T. Yagi, IEEE Trans. Plasma Sci. 25, 1431 (1997).

[53] J.R. Bettis and A.H. Guenther, J. Quan. Elec. 6, 483 (1970).

[54] R. A. Dougal, P. P. Williams, and A. H. Guenther, in 4th IEEE International Pulsed Power Conference, Albuquerque, 1983, Ref. [18], p. 443.

[55] W. K. Pendleton and A. H. Guenther, Rev. Sci. Instrum. 36, 1546 (1965).
[56] W. T. Clark et al., in 16th IEEE Pulsed Power Conference, Albuquerque, NM, 2007, Ref. [10], p. 129.

[57] M.E. Savage, B. S. Stoltzfus, and W. T. Clark (unpublished).

[58] J.M. Wilson and G. L. Donovan, in Proceedings of 6th IEEE International Pulsed Power Conference, Arlington, 1987, Ref. [6], p. 361.

[59] B. N. Turman and D. R. Humphreys, in Proceedings of 6th IEEE International Pulsed Power Conference, Arlington, 1987, Ref. [6], p. 347. 